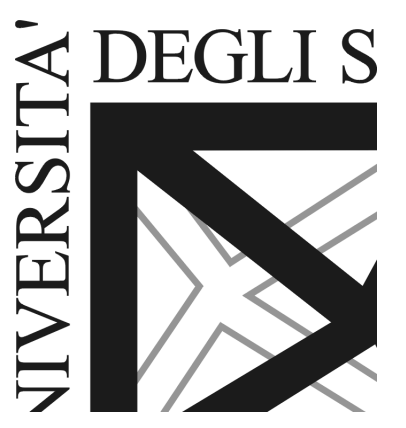

\author{
DEPARTMENT OF ECONOMICS, \\ MANAGEMENT AND STATISTICS \\ UNIVERSITY OF MILAN - BICOCCA
}

DEMS WORKING PAPER SERIES

On the characterization of
quasi-perfect equilibria

Nicola Gatti, Mario Gilli, Alberto Marchesi

No. 389 - November 2018

Dipartimento di Economia, Metodi Quantitativi e Strategie di Impresa Università degli Studi di Milano - Bicocca

http://dems.unimib.it/ 


\title{
ON THE CHARACTERIZATION OF QUASI-PERFECT EQUILIBRIA
}

\author{
Nicola Gatti ${ }^{\mathrm{a}}$, Mario Gilli ${ }^{\mathrm{b}}$ and Alberto Marchesi ${ }^{\mathrm{a}}$
}

Van Damme [1984] introduces the concept of quasi-perfect equilibrium, which refines sequential equilibrium as well as normal-form perfect equilibrium. It has been argued by Mertens [1995] that quasi-perfection is conceptually superior to extensiveform perfection, since quasi-perfection guarantees normal-form perfection, which for two-player games is equivalent to admissibility. On the other hand, while extensiveform perfect equilibria are defined as limit points of sequences of Nash equilibria of a general class of perturbed games in extensive form, till now, to the best of our knowledge, there is no characterization of quasi-perfect equilibria in terms of limit points of equilibria of perturbed games. The only known result is Lemma 1 by Miltersen and Sørensen [2010], showing that limit points of sequences of Nash equilibria of a particular class of perturbed games in sequence form are quasi-perfect equilibria of the original, unperturbed game in extensive form. However, as the authors point out, their main result only proves that a subset of the quasi-perfect equilibria can be obtained as limit points of equilibria of their class of perturbed games, and, thus, their paper provides no characterization of quasi-perfect equilibria in terms of perturbed games. The present paper fills this gap providing such characterization, showing that any quasi-perfect equilibrium can be obtained as limit point of a sequence of Nash equilibria of a certain class of perturbed games in sequence form, at least for the case of two-player games with nature. This result shows that the sequence form is not merely a computationally efficient representation, but it also captures game features that other forms are not able to effectively express.

Keywords: Non-cooperative Game Theory, Extensive Games, Nash Equilibrium Refinements.

Jel Classification: C70, C72.

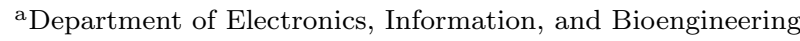
Politecnico di Milano, Via Ponzio, 34/5, I-20133 Milano, Italy

nicola.gatti@polimi.it; alberto.marchesi@polimi.it

${ }^{\mathrm{b}}$ Department of Economics, Management, and Statistics University of Milan-Bicocca, Piazza Ateneo Nuovo 1, 20126 Milan, Italy

mario.gilli@unimib.it 


\section{INTRODUCTION}

Perturbed games and trembles are ubiquitous in the theory of equilibrium refinements. Motivated by the consideration that a player may be more concerned with trembles of others than with her own, Van Damme [1984] introduces the concept of quasi-perfect equilibrium. In a quasi-perfect equilibrium, each player follows a strategy that specifies an optimal choice against trembles of other players at each information set, without taking into account her own trembles, except to the extent that they may make one of her information sets reached that otherwise would not be. According to this definition, trembles encompass the idea that the players should have strategic uncertainty, i.e., they should not be completely confident as to what the other players are going to do. ${ }^{1}$ This leads to some attractive properties of quasi-perfect equilibria. In particular, Van Damme [1984] shows that quasi-perfection refines sequential as well as normal-form perfect equilibria, and that it is neither a refinement nor a broadening of extensiveform perfect equilibria. ${ }^{2}$ Moreover, Mertens [1995] provides an example where the set of quasi-perfect equilibria and that of extensive-form perfect equilibria are disjoint. ${ }^{3}$ Van Damme [1984] also proves that a proper equilibrium of a normal-form game is a quasi-perfect equilibrium in any extensive-form game having that game as normal form. Furthermore, in a quasi-perfect equilibrium, each player plays optimal admissible continuations from every information set of her [Blume et al., 1991]. It has also been argued by Mertens [1995] that quasiperfection is conceptually superior to extensive-form perfection, since there are two-player games where any extensive-form perfect equilibrium involves using a weakly dominated strategy, while quasi-perfection guarantees normal-form perfection, which for two-player games is equivalent to admissibility. This view is shared by Govindan and Wilson, who put quasi-perfection at the center of their axiomatic equilibrium selection theory [Govindan and Wilson, 2012, 2006].

Unfortunately, while extensive-form perfect equilibria are characterized as limit points of sequences of Nash equilibria of perturbed games in extensive form, till now, to the best of our knowledge, there is no characterization of quasi-perfect equilibria in terms of limit points of equilibria of perturbed games. The only known result is Lemma 1 by Miltersen and Sørensen [2010], showing that, limited to two-player games, limit points of sequences of Nash equilibria of a particular class of perturbed games in sequence form are quasi-perfect equilibria of the original, unperturbed game in extensive form. However, as the authors point out, their result only shows that a subset of the quasi-perfect equilibria can be obtained as limit points of Nash equilibria of their class of perturbed games, and, thus, their paper provides no characterization of quasi-perfect equilibria in terms of perturbed games. In this paper, we fill this gap providing such

\footnotetext{
${ }^{1}$ See Hillas and Kohlberg [2002] for additional details.

${ }^{2}$ However, if payoffs are generic, then every sequential equilibrium is quasi-perfect [Hillas et al., 2016, Pimienta and Shen, 2014], and sequential equilibria are also extensive-form perfect equilibria [Blume and Zame, 1994, Kreps and Wilson, 1982].

${ }^{3}$ See also Hillas and Kohlberg [2002].
} 
characterization for two-player games with nature. In particular, we provide a simple example where there is a continuum of quasi-perfect equilibria that are not limit points of sequences of Nash equilibria of perturbed games à la Miltersen and Sørensen. Then, we introduce a general class of perturbed $n$-player games in sequence form generating quasi-perfect equilibria of the original game as limit points of sequences of Nash equilibria. Finally, we show our main result: any quasi-perfect equilibrium of a two-player game in extensive form with nature can be obtained as limit point of a sequence of Nash equilibria of our class of perturbed games in sequence form. In this way, we prove that, in two-player games with nature, quasi-perfect equilibria can be characterized as limit points of Nash equilibria of our class of perturbed games in sequence form. A weaker characterization holds for games with three or more players.

\subsection{Summary of the Results}

In the following, we briefly discuss how we prove our main result. Following the idea of trembles in games in extensive form, we introduce a class of perturbed $n$-player games in sequence form defined by strictly positive lower bounds on the probabilities of playing sequences, such that: (i) lower bounds can be expressed as polynomial functions of the perturbation magnitude; (ii) they approach zero as the perturbation vanishes; and (iii) lower bounds of child sequences approach zero faster than the lower bound of their father sequence. Then, we split the proof of our main result into two steps.

As a first step, we show that any limit point of a sequence of Nash equilibria of our perturbed $n$-player games in sequence form is a quasi-perfect equilibrium of the original, unperturbed game in extensive form. This is a generalization of the result provided by Miltersen and Sørensen [2010], which is limited to two-player games and to a particular class of perturbed games in sequence form (a subclass of ours). Our proof uses different tools from those employed by Miltersen and Sørensen [2010], as their approach cannot be easily generalized to our case. In particular, in order to prove this first part of the result, we use an interesting property of our perturbed games, which is also crucial for the second part of the proof. Specifically, provided that the perturbation magnitude is sufficiently small, Nash equilibria of perturbed games prescribe each player to play optimally everywhere, i.e., also in those parts of the game that are reached with probability zero in absence of perturbation. ${ }^{4}$ Intuitively, this property holds thanks to our definition of perturbed games in sequence form, and condition (iii) in particular. Formally, we prove it by applying the complementarity slackness theorem to the primal-dual pair of linear programming problems for computing a player's best response to the (fixed) opponents' strategies in perturbed games in sequence form. Moreover, this property is strictly connected with the idea of playing an optimal choice against trembles of other players at each information set, which

\footnotetext{
${ }^{4}$ See Theorem 1.
} 
is crucial for the definition of quasi-perfect equilibrium. ${ }^{5}$ This last observation allows us to prove the first part of our main result. ${ }^{6}$

As for the second part of our proof, we show that, given any quasi-perfect equilibrium of a two-player game in extensive form with nature, we can build suitable perturbed games in sequence form, such that they belong to our class and admit a sequence of Nash equilibria having the given quasi-perfect equilibrium as limit point. In order to prove this, we exploit the fact that any quasi-perfect equilibrium can be obtained as limit point of a sequence of completely mixed behavioral strategy profiles described as rational functions. ${ }^{7}$ First, we apply some transformations on these functions in order to build a sequence of completely mixed realization plan profiles described as polynomial functions. ${ }^{8}$ These transformations ensure that the resulting sequence has the given quasi-perfect equilibrium as limit point. Then, we show how to construct perturbed games in sequence form, such that they belong to our class and the sequence of realization plan profiles just described defines a sequence of Nash equilibria for them. ${ }^{9}$ In order to show this, we exploit the property of our perturbed games that we previously discussed. We prove that we can always build such perturbed games, and this is guaranteed by the way we apply the transformations.

\subsection{Structure of the Paper}

The setup of the paper is as follows. Section 2 recalls the notions of game in extensive and in sequence form, and revises the concepts of Nash equilibria and its refinements, stressing the role of perturbations. Section 3 provides an example where there are quasi-perfect equilibria that are not limit points of sequences of Nash equilibria of games perturbed à la Miltersen and Sørensen, motivating a general discussion on the relationship between perturbed games in extensive and in sequence form, and the introduction of an alternative definition of quasiperfect equilibrium, following Van Damme [1984]. Section 4 introduces our class of $n$-player perturbed games in sequence form and studies the properties of Nash equilibria in these perturbed games, providing the conceptual tools required by Sections 5 and 6 , which are the core of the paper. Section 5 proves that limit points of sequences of Nash equilibria of our class of $n$-player perturbed games in sequence form are quasi-perfect equilibria of the original games (a generalization of the Miltersen and Sørensen [2010] result), while Section 6 proves that quasiperfect equilibria of two-player games with nature are limit points of sequences of Nash equilibria of our class of perturbed games in sequence form, completing the characterization. Section 7 concludes the paper arguing for the expressiveness of the sequence form.

\footnotetext{
${ }^{5}$ See Lemma 4.

${ }^{6}$ See Theorem 2.

${ }^{7}$ See Remark 2.

${ }^{8}$ See Definitions 22 and 25.

${ }^{9}$ See Definition 19, Lemmas 5 and 6, and Theorem 3.
} 


\section{PRELIMINARIES}

In this section, we review notation and definitions required for the results provided in the following sections. First, we introduce the fundamental notions related to games in extensive and sequence form. Then, we revise the definitions of Nash equilibrium and some of its refinements, specifically extensive-form perfect and quasi-perfect equilibria.

\subsection{Games in Extensive Form}

In this subsection, we state our notation and conventions for finite games in extensive form, following Selten [1975] and Von Stengel [1996]. The basic structure of a game in extensive form is the game tree $T$, which is a finite, directed tree with a distinguished node, the root denoted by $o$, from which there is a unique path to every other node. Edges of the tree are denoted by $x y$, where $x$ and $y$ are the nodes defining the endpoints of the edge, and $y$ is a child of $x$. Nodes without children are called leaves (or terminal nodes, or outcomes) and their set is denoted by $Z$, the others are decision nodes, and their set is denoted by $X$. The set of all nodes is $K=X \cup Z$.

In addition to the game tree $T$, a game in extensive form has the following components. There are $n$ players numbered from 1 to $n$, with $N=\{1, \ldots, n\}$ denoting the set of players. Moreover, the game has also random chance moves that are usually treated as if they are played by an additional player, called chance player and denoted as player 0 . A further element of a game in extensive form is the player function $\iota: X \rightarrow N \cup\{0\}$ that assigns to each decision node the player whose turn it is. Using this function, the set of decision nodes $X$ can be partitioned in $X_{0}, X_{1}, \ldots, X_{n}$ so that each element $x \in X_{i}$ belongs to exactly one player $i \in N \cup\{0\}$, called the player to move at $x$, i.e., $\iota(x)=i$ for all $x \in X_{i}$. The set of decision nodes $X$ is partitioned into information sets. Each information set $u$ belongs to exactly one player $i \in N \cup\{0\}$, called the player to move at $u$, i.e., $\iota(x)=i$ for all $x \in u$. The set of all information sets of player $i$ is denoted by $U_{i}$, while $U=\bigcup_{i \in N \cup\{0\}} U_{i}$ is the set of all information sets in the game. The idea is that for any $x, x^{\prime} \in u \in U_{i}$, player $i$ is not able to distinguish $x$ from $x^{\prime}$, i.e., the path connecting the root $o$ to $x$ from the path connecting $o$ to $x^{\prime}$.

The possible moves of a player are represented by a function that assigns to each edge $x y$ a label, called a choice at $x$, such that the choices at $x$ are always distinct, i.e., the children $y$ of a decision node $x$ can be distinguished by the respective labels of the edges $x y$. Since a player is not able to distinguish the nodes in the same information set, for all nodes $x \in u$ there are the same possible choices, called the choices at $u$, and the set of these choices is denoted by $C_{u}$. In particular, all nodes $x \in u$ have the same number $\left|C_{u}\right|$ of children. For simplicity, it is assumed that for any pair of information sets $u, v \in U$, the choice sets $C_{u}$ and $C_{v}$ are disjoint. Finally, let us denote by $D_{i}=\bigcup_{u \in U_{i}} C_{u}$ the set of all choices 
of player $i$, and by $D=\bigcup_{u \in U} C_{u}$ the set of all choices in the game.

We say that a decision node $x \in K$ precedes a node $y \in K$ if there is a path from $x$ to $y$, and that $y \in K$ is reachable from $u \in U$ if there is some node $x \in u$ preceding $y$. The sequence of choices assigned to edges on the path connecting the root $o$ with a vertex $x \in K$ is called the path to $x$, and it is denoted by $p(x)$. A path to a leaf $z \in Z$ is called a play.

In this work, each player is assumed to have perfect recall. This is a structural property of the information sets of the player, reflecting the hypothesis that she does not forget what she knew or did earlier. The meaning is that a player who has to make a decision at one of her information sets knows which of her other information sets have been reached by the previous course of the play and which choices have been taken there. In games with perfect recall, we can define a precedence relation over information sets of the same player, following Von Stengel and Forges [2008]. Formally, for any player $i \in N \cup\{0\}, u \in U_{i}$ is said to precede $v \in U_{i}$, denoted by $u \preceq v$, if there is a node $y \in v$ that is reachable from $u$. We also write $u \prec v$ if $u \preceq v$ and $u \neq v$. Moreover, it is possible to prove Kuhn theorem [Kuhn, 1953], which states that, in the context of games in extensive form with perfect recall, instead of using mixed strategies, one can restrict attention to behavior strategies without loss of generality. ${ }^{10}$

For player $i \in N \cup\{0\}$, a behavior strategy $\beta_{i} \in B_{i}$ is a probability distribution on $C_{u}$ for each information set $u \in U_{i}$, formally it is a function $\beta_{i}: D_{i} \rightarrow \mathbb{R}^{+}$such that $\sum_{c \in C_{u}} \beta_{i}(c)=1$ for all $u \in U_{i}$. Fixed positive probabilities for the chance moves are also part of the game in extensive form, and they can be specified as a behavior strategy $\beta_{0}$ for player 0 . Without loss of generality, for any $c \in D_{0}$, we assume $\beta_{0}(c)>0$, since branches of the game tree that are never reached can be pruned. A behavior strategy profile is a tuple $\beta=\left(\beta_{i}\right)_{i \in N \cup\{0\}}=\left(\beta_{-i}, \beta_{i}\right) \in B$, where $\beta_{-i}$ denotes the partial strategy profile obtained by removing player $i$ 's strategy from $\beta$. We say that $\beta \in B$ is completely mixed if, for any $i \in N$, every behavior strategy $\beta_{i}$ assigns strictly positive probability to every choice $c \in D_{i}$. Moreover, $\left(\beta / \beta_{i}^{\prime}\right)$ denotes the behavior strategy profile obtained from $\beta=\left(\beta_{-i}, \beta_{i}\right) \in B$ by replacing player $i$ 's behavior strategy $\beta_{i}$ with $\beta_{i}^{\prime} \in B_{i}$.

Finally, for $i \in N$, player $i$ 's payoff function $h_{i}$ is defined on the set of leaves $Z$, i.e., $h_{i}: Z \rightarrow \mathbb{R}$. We remark that chance receives no payoff. Moreover, we can also define the payoff vector function $h: Z \rightarrow \mathbb{R}^{n}$, which assigns a vector of players' payoffs to each leaf, i.e., $h$ is a vector-valued function whose $i$-th component is the scalar function $h_{i}$.

Next, we introduce the formal definition of finite game in extensive form, as stated by Selten [1975].

Definition 1 A game in extensive form is a tuple:

$$
\Gamma=\left(T, N, \iota, U, D, \beta_{0}, h\right)
$$

\footnotetext{
${ }^{10}$ See Selten [1975].
} 
where:

- $T$ is the finite game tree;

- $N=\{1, \ldots, n\}$ is the set of players;

- $\iota: X \rightarrow N \cup\{0\}$ is the player function;

- $U$ is the information partition;

- $D$ is the set of possible choices;

- $\beta_{0}$ is the fixed positive probability distribution for the chance moves;

- $h$ is the payoff vector function.

Using the elements of a game in extensive form, we can define some further concepts. For any behavior strategy profile $\beta \in B$, it is immediate to define the probability of reaching any node $x \in K$, denoted by $\mathbb{P}(x \mid \beta)$, by just multiplying the probability of the choices on the path from the root $o$ to $x$, formally: $\mathbb{P}(x \mid \beta)=$ $\prod_{j \in N \cup\{0\}} \prod_{c \in p(x) \cap D_{j}} \beta_{j}(c)$. Additionally, for any information set $u \in U$, we can define $\mathbb{P}(u \mid \beta)$ as the sum of the probabilities of reaching the nodes in $u$ when $\beta$ is played.

For $i \in N$, player $i$ 's expected payoff associated to a strategy profile $\beta \in B$ is defined as $H_{i}(\beta)=\sum_{z \in Z} \mathbb{P}(z \mid \beta) h_{i}(z)$. Furthermore, letting $Z(u) \subseteq Z$ be the set of leaves reachable from $u \in U$, for any $\beta \in B$, if $\mathbb{P}(u \mid \beta)>0$, then it is possible to define the player $i$ 's expected payoff conditional to an information set $u \in U_{i}$ being reached, as follows:

$$
H_{i}(\beta \mid u)=\sum_{z \in Z(u)} \frac{\mathbb{P}(z \mid \beta)}{\mathbb{P}(u \mid \beta)} h_{i}(z) .
$$

Table I sums up the main symbols used for games in extensive form.

\subsection{Games in Sequence Form}

Von Stengel [1996] defines the sequence form for any game in extensive form with perfect recall. The sequence form is a strategic description which represents strategies in a different way with respect to the extensive form: rather than as complete plans of moves at every information set, a player looks at each leaf of the game tree and considers the sequence of choices she needs to make so that the leaf can be reached. These are the player's choices prescribed by the respective play, i.e., the path from the root $o$ to the leaf. They represent a "sequence" that will be considered instead of a pure strategy. Formally, any node $x \in K$ of the game tree, for any player $i \in N \cup\{0\}$, defines a sequence $s_{i}$ of choices in $D_{i}$ on the path from the root $o$ to $x$, i.e., choices in $D_{i} \cap p(x)$. The set of all sequences of player $i$ is denoted by $S_{i}$, while a sequence profile is a tuple $\left(s_{0}, s_{1}, \ldots, s_{n}\right) \in S_{0} \times S_{1} \times \ldots \times S_{n}=S$. Notice that a sequence $s_{i}$ can be regarded as a string of player $i$ 's choices found on the path to some node $x$. However, for easy reference to its elements, we can define sequence $s_{i}$ as the set of its choices. This is possible since we assumed that choices at different 


\begin{tabular}{|c|c|}
\hline Symbol & Meaning \\
\hline$T$ & game tree \\
\hline$o$ & root of the game tree \\
\hline$x y$ & edge of the tree, $y$ child of $x$ \\
\hline$Z$ & terminal nodes of the game tree \\
\hline$X$ & decision nodes of the game tree \\
\hline$K=X \cup Z$ & nodes of the game tree \\
\hline$N=\{1, \ldots, n\}$ & players \\
\hline 0 & chance player \\
\hline$\iota: X \rightarrow N \cup\{0\}$ & $\begin{array}{c}\text { function that specifies the player } \\
\text { whose turn is to choose at each decision node }\end{array}$ \\
\hline$u \in U_{i}$ & information set of player $i$ \\
\hline$C_{u}$ & set of choices at $u$ \\
\hline$D_{i}$ & set of all choices of player $i$ \\
\hline$\beta_{i} \in B_{i}$ & player $i$ 's behavior strategy \\
\hline$\beta=\left(\beta_{-i}, \beta_{i}\right) \in B$ & behavior strategy profile \\
\hline$\beta / \beta_{i}^{\prime}$ & $\begin{array}{c}\text { behavior strategy profile obtained from } \beta \in B \\
\text { by replacing } \beta_{i} \text { with } \beta_{i}^{\prime} \in B_{i}\end{array}$ \\
\hline $\mathbb{P}(x \mid \beta)$ & probability of reaching $x \in K$ if $\beta \in B$ is played \\
\hline$h_{i}: Z \rightarrow \mathbb{R}$ & player $i$ 's payoff function \\
\hline$H_{i}(\beta)=\sum_{z \in Z} \mathbb{P}(z \mid \beta) h_{i}(z)$ & player $i$ 's expected payoff associated to $\beta \in B$ \\
\hline$H_{i}(\beta \mid u)=\sum_{z \in Z(u)} \frac{\mathbb{P}(z \mid \beta)}{\mathbb{P}(u \mid \beta)} h_{i}(z)$ & $\begin{array}{c}\text { player } i \text { 's expected payoff conditional to } u \in U_{i} \\
\text { being reached associated to } \beta \in B\end{array}$ \\
\hline$p(x)$ & path to a node $x \in K$ \\
\hline$p(z)$ & path to a leaf $z \in Z$, i.e., a play \\
\hline$u \preceq v$ & $u \in U_{i}$ precedes $v \in U_{i}$ \\
\hline$u \prec v$ & $u \in U_{i}$ precedes $v \in U_{i}$ and $u \neq v$ \\
\hline
\end{tabular}

TABLE I

NOTATION FOR GAMES IN EXTENSIVE FORM 
information sets are distinct. Note that, for each player, there are at most as many sequences as the number of nodes of the game tree; so their number is linear in the size of the game tree, in contrast to the number of pure strategies which may be exponential in the size of the tree.

By definition of perfect recall, every node in an information set $u \in U_{i}$ defines the same sequence of choices for player $i$, hence the following definitions are well posed. For $i \in N \cup\{0\}$, the function $\sigma: U_{i} \rightarrow S_{i}$ returns the sequence $s_{i} \in S_{i}$ defined by nodes in $u \in U_{i}$. For any $u \in U$ and $c \in C_{u}$, the sequence $\sigma(u)$ can be extended by adding $c$ at the end, i.e., $\sigma(u) c=\sigma(u) \cup c$. Thus, the set $S_{i}$ of player $i$ 's sequences can be represented as $S_{i}=\{\varnothing\} \cup\left\{\sigma(u) c \mid u \in U_{i}, c \in C_{u}\right\}$. Note that $\sigma(o)=\varnothing$, hence the empty sequence $\varnothing$ belongs to $S_{i}$, for any $i \in N \cup\{0\}$. To simplify notation, given a sequence $s_{i} \in S_{i}$ and a choice $c \in D_{i}$, we denote as $s_{i} \mid c$ the extended sequence obtained by appending $c$ to $s_{i}$ (if the resulting sequence is well-defined for player $i$ ). Moreover, for every pair of sequences $s_{i}, s_{i}^{\prime} \in S_{i}$, we write $s_{i}^{\prime} \sqsubset s_{i}$ whenever the set of choices defining sequence $s_{i}^{\prime}$ is a strict subset of that defining sequence $s_{i}$. The sequences of the chance player 0 are also considered, since it is treated as the other players.

In order to define the payoffs associated to sequence profiles, note that, by definition of game tree, there is a unique path from the root $o$ to every other node, hence for any node $x \in K$ there exists a unique sequence profile $\left(s_{0}, s_{1}, \ldots, s_{n}\right) \in S$ leading to $x$. Hence, we can define the function $\xi: K \rightarrow S$ specifying the sequence profile reaching $x \in K$. Then, in a game in sequence form, the player $i$ 's payoff function is $g_{i}: S \rightarrow \mathbb{R}$, where:

$$
g_{i}\left(s_{0}, s_{1}, \ldots, s_{n}\right)= \begin{cases}h_{i}(z) & \text { if }\left(s_{0}, s_{1}, \ldots, s_{n}\right)=\xi(z) \\ 0 & \text { otherwise }\end{cases}
$$

Then, $g=S \rightarrow \mathbb{R}^{n}$ is the payoff vector function of the game in sequence form, where the $i$-th component of $g$ is function $g_{i}$.

In addition to the payoffs, it is also necessary to specify how sequences are selected by a player. In games in extensive form, because of Kuhn Theorem, a player may just decide by a behavior strategy. In games in sequence form, instead, a player cannot just decide on a single sequence. In general, behavior strategy probabilities will be replaced by the realization probabilities of sequences. For any player $i \in N \cup\{0\}$, the realization plan of a behavior strategy $\beta_{i} \in B_{i}$ is the function $r_{i}: S_{i} \rightarrow \mathbb{R}$ defined as follows: for any sequence $s_{i} \in S_{i}$, it holds $r_{i}\left(s_{i}\right)=\prod_{c \in s_{i}} \beta_{i}(c)$. Then, for any information set $u \in U_{i}$ and choice $c^{\prime} \in C_{u}$, we have $r_{i}\left(\sigma(u) c^{\prime}\right)=\prod_{c \in \sigma(u)} \beta_{i}(c) \beta_{i}\left(c^{\prime}\right)$. By construction, a realization plan of a behavior strategy can be characterized by the following linear restrictions:

$$
r_{i}(\varnothing)=1,
$$

because the empty product is 1 ; for any information set $u \in U_{i}$

$$
-r_{i}(\sigma(u))+\sum_{c \in C_{u}} r_{i}(\sigma(u) c)=0
$$


because $\sum_{c \in C_{u}} \beta_{i}(c)=1$; and for every sequence $s_{i} \in S_{i}$

$$
r_{i}\left(s_{i}\right) \geq 0,
$$

because it is a probability. This leads to the following definition: a function $r_{i}: S_{i} \rightarrow \mathbb{R}^{+}$fulfilling restrictions (1), (2), and (3) is a realization plan for player $i \in N \cup\{0\}$. The set of all realization plans for player $i$ is denoted by $R_{i}$, while $r=\left(r_{i}\right)_{i \in N \cup\{0\}}=\left(r_{-i}, r_{i}\right) \in R$ is a tuple representing a realization plan profile. Moreover, we use $\left(r / r_{i}^{\prime}\right)$ to denote the realization plan profile obtained from $r \in R$ by replacing player $i$ 's realization plan with $r_{i}^{\prime} \in R_{i}$.

Let us recall that Von Stengel [1996] proves that any realization plan $r_{i}: S_{i} \rightarrow$ $\mathbb{R}^{+}$satisfying linear restrictions (1), (2), and (3) arises from a suitable behavior strategy such that $\beta_{i}(c)=\frac{r_{i}(\sigma(u) c)}{r_{i}(\sigma(u))}$ for all $u \in U_{i}$ and $c \in C_{u}$ if $r_{i}(\sigma(u))>0$, while $\beta_{i}(c)$ can be any otherwise.

Now, it is possible to provide the formal definition of the game in sequence form associated to a finite game in extensive form with perfect recall.

DeFINITION 2 The game in sequence form associated to a finite game in extensive form with perfect recall $\Gamma$ is the tuple:

$$
\Gamma_{S}=\left(S, N, R, r_{0}, g\right),
$$

where:

- $S=S_{0} \times S_{1} \times \ldots \times S_{n}$ is the set of sequence profiles;

- $N=\{1, \ldots, n\}$ is the set of players;

- $R=R_{0} \times R_{1} \times \ldots \times R_{n}$ is the set of realization plan profiles, where each $R_{i}$ is the set of player $i$ 's realization plans described by the linear restrictions (1), (2), and (3);

- $r_{0}$ is the realization plan of $\beta_{0}$;

- $g$ is the payoff vector function of the sequence form.

As for games in extensive form, for any $i \in N$, we define the player $i$ 's expected payoff associated to a realization plan profile $r \in R$, as follows:

$$
G_{i}(r)=\sum_{s \in S} g_{i}(s) \prod_{i \in N \cup\{0\}} r_{i}\left(s_{i}\right) .
$$

When working with the sequence form, it is useful to adopt matrix notation. In particular, each function $g_{i}$ can be described as a multi-dimensional matrix $M_{i}$ indexed by sequence profiles $s=\left(s_{0}, s_{1}, \ldots, s_{n}\right) \in S$, such that the entry of $M_{i}$ corresponding to $s \in S$ is equal to $g_{i}(s)$. Moreover, we can overload the definition of $r_{i}$ and use it as a nonnegative vector with $\left|S_{i}\right|$ components representing a realization plan. Then, we can express linear restrictions (1), (2), and (3) by a "constraint" matrix $F_{i}$ with $1+\left|U_{i}\right|$ rows and $\left|S_{i}\right|$ columns, such that vectors $r_{i}$ must satisfy the following matrix equality:

$$
F_{i} r_{i}=f_{i}
$$


ON THE CHARACTERIZATION OF QUASI-PERFECT EQUILIBRIA

\begin{tabular}{|c|c|}
\hline Symbols & Meaning \\
\hline$s_{i} \in S_{i}$ & sequence of player $i$ \\
\hline$\sigma: U_{i} \rightarrow S_{i}$ & function defining sequence $s_{i}$ leading to $u \in U_{i}$ \\
\hline$\sigma(u) c$ & sequence leading to $u$ extended by $c \in C_{u}$ \\
\hline$\xi: K \rightarrow S$ & function defining the sequence profile reaching $x \in K$ \\
\hline$g_{i}: S \rightarrow \mathbb{R}$ & player $i$ 's payoff function in the sequence form \\
\hline$M_{i}$ & player $i$ 's payoff matrix \\
\hline$r_{i}: S_{i} \rightarrow \mathbb{R}^{+}$ & player $i$ 's realization plan \\
\hline$F_{i} r_{i}=f_{i}$ & player $i$ 's expected payoff associated to $r \in R$ \\
\hline$g_{i}(r)=\sum_{i \in S} g_{i}(s) r_{j}\left(s_{j}\right)$ & \\
\hline$s_{i} \mid c$ & extended sequence obtained by appending $c$ to $s_{i}$ \\
\hline$s_{i}^{\prime} \sqsubset s_{i}$ & sequence $s_{i}^{\prime}$ is a strict subset of sequence $s_{i}$ \\
\hline
\end{tabular}

TABLE II

NotATION FOR GAMES IN SEQUENCE FORM

where $f_{i}$ is a vector with $1+\left|U_{i}\right|$ components such that $f_{i}=(1,0, \ldots, 0)^{T}$. Finally, using matrix notation, we can write:

$$
G_{i}(r)=r_{i}^{T}\left(M_{i} \prod_{j \neq i \in N \cup\{0\}} r_{j}\right),
$$

assuming the product involving vectors $r_{j}$ is appropriately defined.

Table II sums up the notation used for games in sequence form.

\subsection{Nash Equilibria in Games in Extensive Form and in Sequence Form}

Formally, in a game in extensive form we have the following definition.

Definition $3 \quad A$ behavior strategy profile $\beta^{*} \in B$ is a Nash equilibrium of a game in extensive form $\Gamma$, denoted by $\beta^{*} \in N E(\Gamma)$, if and only if for any player $i \in N$ it holds:

$$
\beta_{i}^{*} \in \underset{\beta_{i} \in B_{i}}{\arg \max } H_{i}\left(\beta^{*} / \beta_{i}\right)
$$

Similarly, in a game in sequence form we have the following definition, which is equivalent to the previous one, given the relationship between extensive form and sequence form in games with perfect recall.

Definition $4 A$ realization plan profile $r^{*} \in R$ is a Nash equilibrium of a game in sequence form $\Gamma_{\boldsymbol{S}}$, denoted by $r^{*} \in N E\left(\Gamma_{\boldsymbol{S}}\right)$, if and only if for any player $i \in N$ it holds:

$$
r_{i}^{*} \in \underset{r_{i} \in R_{i}}{\arg \max } G_{i}\left(r^{*} / r_{i}\right)
$$




\subsection{Extensive-Form Perfect Equilibria}

Selten [1975] has been the first game theorist proposing perturbed games to model equilibrium refinements. The original idea of Selten was that, however close to being rational players were, they would never be perfectly rational. Thus, there would always be some chance that a player would make a mistake. This idea may be implemented by approximating a candidate equilibrium strategy profile by a nearby completely mixed strategy profile and requiring that any of the deliberately chosen actions, i.e., those given positive probability in the candidate strategy profile, be optimal, not only against the candidate strategy profile, but also against the nearby completely mixed strategy profile.

Formally, we consider the definition of extensive-form perfect equilibrium provided by Van Damme [1984].

DEFINITION 5 In a game in extensive form $\Gamma$, a behavior strategy profile $\beta^{\varepsilon} \in B$ is an $\varepsilon$-perfect equilibrium of $\Gamma$, if and only if it is fully mixed, and for each player $i \in N$, for all $u \in U_{i}$ and $c, c^{\prime} \in C_{u}$, it holds:

$$
H_{i}\left(\beta^{\varepsilon} / c \mid u\right)<H_{i}\left(\beta^{\varepsilon} / c^{\prime} \mid u\right) \Longrightarrow \beta_{i}^{\varepsilon}(c) \leq \varepsilon,
$$

where, for any $c \in C_{u},\left(\beta^{\varepsilon} / c\right)$ denotes the strategy profile obtained from $\beta^{\varepsilon}$ by letting player $i$ play choice $c$ with probability one at $u$.

DEFINITION 6 In a game in extensive form $\Gamma$, a behavior strategy profile $\beta^{*} \in B$ is an extensive-form perfect equilibrium of $\Gamma$, denoted by $\beta^{*} \in P E(\Gamma)$, if and only if it is a limit point (as $\varepsilon$ goes to zero) of $\varepsilon$-perfect equilibria $\beta^{\varepsilon} \in B$ of $\Gamma$.

Now, we introduce the concepts proposed by Selten [1975].

DeFinition 7 For any game in extensive form $\Gamma, a$ Selten perturbed game in extensive form $\Gamma^{S e}$ is a pair $(\Gamma, \eta)$ where $\eta$ is a function

$$
\eta: D \rightarrow(0,1)
$$

assigning a positive lower bound on the probability of playing each choice $c \in D$, such that, for any information set $u \in U$, it holds $\sum_{c \in C_{u}} \eta(c)<1$.

Lower bounds on the probabilities of playing choices represent what are usually referred to as trembles, which model the idea that players are not perfectly rational and, thus, they can make mistakes.

Definition 8 For any game in extensive form $\Gamma$, a Selten test sequence is a sequence of Selten perturbed games in extensive form $\left\{\left(\Gamma, \eta_{k}\right)\right\}_{k \in \mathbb{N}}$ such that for any information set $u \in U$ and choice $c \in C_{u}$ it holds:

$$
\lim _{k \rightarrow \infty} \eta_{k}(c)=0 .
$$


DEFINITION 9 In a game in extensive form $\Gamma$, a behavior strategy profile $\beta^{*} \in B$ is a limit equilibrium of a Selten test sequence $\left\{\left(\Gamma, \eta_{k}\right)\right\}_{k \in \mathbb{N}}$ if there exists a sequence of behavior strategy profiles $\left\{\beta_{k}\right\}_{k \in \mathbb{N}}$ such that each $\beta_{k} \in B$ is a Nash equilibrium of $\left(\Gamma, \eta_{k}\right)$ and $\beta^{*}$ is a limit point of the sequence.

Finally, from Lemma 5 and Theorem 4 of Selten [1975], it follows the characterization of extensive-form perfect equilibria in terms of Selten perturbed games in extensive form.

Proposition 1 In a game in extensive form $\Gamma$, a behavior strategy profile $\beta^{*} \in B$ is an extensive-form perfect equilibrium of $\Gamma$ if and only if it is a limit equilibrium of some Selten test sequence $\left\{\left(\Gamma, \eta_{k}\right)\right\}_{k \in \mathbb{N}}$.

\subsection{Quasi-Perfect Equilibria}

From the previous definitions, it is clear that, in an extensive-form perfect equilibrium, each player takes into account the possibility that both herself and her opponents may tremble in future. On the other hand, in a quasi-perfect equilibrium, introduced by Van Damme [1984], players only consider the possibility of opponents' trembles. On one hand, the definition of extensive-form perfect equilibrium may be thought of as corresponding to the idea that players really do make mistakes. On the other hand, one might think of the trembles as instead encompassing the idea that the players should have a little strategic uncertainty, i.e., they should not be completely confident as to what the other players are going to do. In such a case a player should not be thought of as being uncertain about her own actions or planned actions. This is a possible interpretation of the idea behind van Damme's definition of quasi-perfect equilibrium.

Van Damme [1984] and Miltersen and Sørensen [2010] present several definitions of quasi-perfect equilibria. We focus, here, on the definition used by Miltersen and Sørensen [2010]. In order to do that, we need some preliminary definitions.

Definition $10 A u$-local purification of a behavior strategy $\beta_{i} \in B_{i}$ is a player $i$ 's behavior strategy $\beta_{i}^{u P} \in B_{i}^{u P}\left(\beta_{i}\right)$ such that:

- $\beta_{i}^{u P}$ is identical to $\beta_{i}$ at all $v \in U_{i}$ such that $v \prec u$;

- for all $v \in U_{i}$ such that $u \preceq v$, there is a choice $c \in C_{v}$ with $\beta_{i}^{u P}(c)=1$.

Definition 11 A u-local purification of $\beta_{i} \in B_{i}$, say $\beta_{i}^{u P^{\prime}} \in B_{i}^{u P}\left(\beta_{i}\right)$, is a $u$-local best response to $\beta \in B$ if and only if it achieves the best expected payoff against $\beta$ among all $u$-local purifications of $\beta_{i}$, i.e., it holds:

$$
\beta_{i}^{u P^{\prime}} \in \underset{\beta_{i}^{u P} \in B_{i}^{u P}\left(\beta_{i}\right)}{\arg \max } H_{i}\left(\beta / \beta_{i}^{u P}\right) .
$$


Definition $12 A$ u-local purification of $\beta_{i} \in B_{i}$, say $\beta_{i}^{u P^{\prime}} \in B_{i}^{u P}\left(\beta_{i}\right)$, is $\varepsilon$ consistent with $\beta_{i}$, denoted by $\beta_{i}^{u P^{\prime}} \in B_{i}^{\varepsilon C}\left(\beta_{i}^{u}\right)$, if and only if, at all information sets $v \in U_{i}$ such that $u \preceq v, \beta_{i}$ assigns probability strictly bigger than $\varepsilon$ to all choices to which $\beta_{i}^{u P^{\prime}}$ assigns probability one.

DEFINITION 13 In a game in extensive form $\Gamma$, a behavior strategy profile $\beta^{\varepsilon} \in$ $B$ is an $\varepsilon$-quasi-perfect equilibrium of $\Gamma$ if and only if it is fully mixed, and for each player $i \in N$ and for any $u \in U_{i}$, all $u$-local purifications of $\beta_{i}^{\varepsilon}$ that are $\varepsilon$-consistent with $\beta_{i}^{\varepsilon}$ are $u$-local best responses to $\beta^{\varepsilon}$. Formally, $\beta^{\varepsilon} \in \varepsilon Q P(\Gamma)$ if and only if, for all $i \in N$ and for any $u \in U_{i}$, it holds:

1. $\beta_{i}^{\varepsilon}(c)>0$ for every choice $c \in C_{u}$;

2. $B_{i}^{\varepsilon C}\left(\beta_{i}^{\varepsilon, u}\right) \subseteq \underset{\beta_{i}^{u P} \in B_{i}^{u P}\left(\beta_{i}^{\varepsilon}\right)}{\arg \max } H_{i}\left(\beta^{\varepsilon} / \beta_{i}^{u P}\right)$.

DEFINITION 14 In a game in extensive form $\Gamma$, a behavior strategy profile $\beta^{*} \in$ $B$ is a quasi-perfect equilibrium of $\Gamma$, denoted by $\beta^{*} \in Q P(\Gamma)$, if and only if it is a limit point (as $\varepsilon$ goes to zero) of $\varepsilon$-quasi-perfect equilibria $\beta^{\varepsilon} \in B$ of $\Gamma$.

Next, we introduce the perturbed games in sequence form proposed by Miltersen and Sørensen [2010]. They are based on the idea of forcing a lower bound on the realization probability of each sequence, where the magnitude of the lower bound depends on the length of the sequence.

Definition 15 For any game in extensive form $\Gamma$, a Miltersen-Sørensen perturbed game $\Gamma^{M S}$ is a pair $\left(\Gamma_{\boldsymbol{S}}, \varepsilon\right)$ where $\varepsilon \in(0,1)$ and for any player $i \in N$, for any realization plan $r_{i} \in R_{i}$, and for all $s_{i} \in S_{i}$ it holds:

$$
r_{i}\left(s_{i}\right) \geq \varepsilon^{\left|s_{i}\right|},
$$

where $\left|s_{i}\right|$ is the number of choices in the sequence $s_{i}$.

Then, Miltersen and Sørensen [2010] prove the following result.

Proposition 2 In a two-player game in extensive form $\Gamma$, for any $\varepsilon>0$ :

$$
N E\left(\Gamma^{M S}\right) \subseteq \varepsilon Q P(\Gamma) .
$$

In words, for two-player games in extensive form, Miltersen and Sørensen [2010] show that a limit point of a sequence of Nash equilibria of their perturbed games in sequence form is a quasi-perfect equilibrium of the original, unperturbed game. However, there are quasi-perfect equilibria that cannot be characterized in this way, as shown in the following section. 


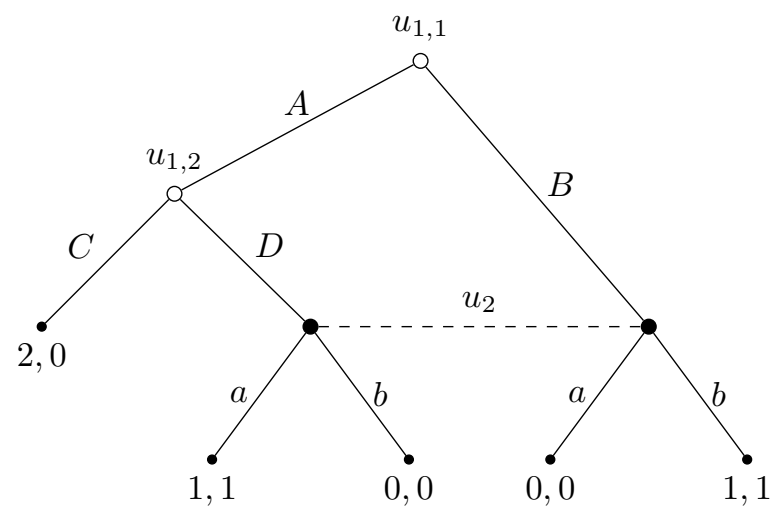

Figure 1.- Example of game in extensive form.

\section{REMARKS ON PERTURBED GAMES AND REFINEMENTS OF NASH EQUILIBRIA}

This section prepares for the core results in this paper, which are the lemmas and theorems in Sections 4, 5, and 6. First, we provide an example showing that there are quasi-perfect equilibria that are not captured by Miltersen-Sørensen perturbed games. This motivates the introduction of an alternative definition of quasi-perfect equilibrium, following Van Damme [1984], and a general discussion on the relationship between perturbed games in extensive and in sequence form.

\subsection{A Motivating Example}

The game in extensive form of Figure 1 shows that the result of Miltersen and Sørensen [2010] (see also Proposition 2) does not provide a characterization of quasi-perfect equilibria in terms of perturbed games, because there are quasiperfect equilibria that are not limit points of sequences of Nash equilibria of Miltersen-Sørensen perturbed games in sequence form. ${ }^{11}$

In particular, in the game of Figure 1, the sets of rationalizable strategies, of Nash equilibria, of normal-form perfect equilibria, of extensive-form perfect equilibria, and of quasi-perfect equilibria coincide and they are:

$$
\left\{\beta_{1} \in B_{1}: \beta_{1}(A)=\beta_{1}(C)=1\right\} \times B_{2} .
$$

However, note that for any Miltersen-Sørensen perturbed game $\Gamma^{M S}$ :

$$
r_{1}(B) \geq \varepsilon \text { and } r_{2}(A D) \geq \varepsilon^{2},
$$

\footnotetext{
${ }^{11}$ In the game of Figure 1 all quasi-perfect equilibria result in the same outcome. However, there are games with outcomes identified by quasi-perfect equilibria that cannot be obtained as limit points of Nash equilibria of Miltersen-Sørensen perturbed games. Some examples are available form authors at request.
} 
which implies that, in any Nash equilibrium of the Miltersen-Sørensen perturbed game, action $b$ is played with probability $1-\varepsilon$ by the second player, hence:

$$
\begin{aligned}
N E\left(\Gamma^{M S}\right)= & \left\{\beta_{1} \in B_{1}: \beta_{1}(A)=1-\varepsilon, \beta_{1}(C)=\frac{1-\varepsilon-\varepsilon^{2}}{1-\varepsilon}\right\} \times \\
& \times\left\{\beta_{2} \in B_{2}: \beta_{2}(b)=1-\varepsilon\right\} .
\end{aligned}
$$

Clearly, this shows that there is a continuum of $\varepsilon$-quasi-perfect equilibria that are not Nash equilibria of $\Gamma^{M S}$, namely all those with $\beta_{2}(a)>0$ as $\varepsilon$ goes to zero. Hence:

REMARK 1 There exist games in extensive form $\Gamma$ such that:

$$
N E\left(\Gamma^{M S}\right) \subset \varepsilon Q P(\Gamma) .
$$

\subsection{An Alternative Definition of Quasi-Perfect Equilibria}

In order to characterize quasi-perfect equilibria, we need to generalize in an opportune way Miltersen-Sørensen perturbed games. In particular, in order to prove our result, we resort to an alternative definition of quasi-perfect equilibria, following one of those provided by Van Damme [1984].

First, we need some auxiliary notation and definitions. Given two behavior strategies $\beta_{i}, \beta_{i}^{\prime} \in B_{i}$ and an information set $u \in U_{i}$, we write $\beta_{i} \underset{u}{=} \beta_{i}^{\prime}$ whenever $\beta_{i}(c)=\beta_{i}^{\prime}(c)$ for every choice $c \in C_{u}$. Furthermore, given $\beta_{i}, \beta_{i}^{\prime} \in B_{i}$ and $u \in U_{i}$, we write $\beta_{i} /{ }_{u} \beta_{i}^{\prime}$ to denote a behavior strategy which is equal to $\beta_{i}^{\prime}$ at all information sets $v \in U_{i}$ such that $u \preceq v$, while it is equal to $\beta_{i}$ at the other information sets. In addition, overloading notation, given $\beta \in B, \beta_{i}^{\prime} \in B_{i}$, and $u \in U_{i}, \beta /{ }_{u} \beta_{i}^{\prime}$ denotes a strategy profile obtained from $\beta$ by replacing player $i$ 's strategy $\beta_{i}$ with $\beta_{i} /{ }_{u} \beta_{i}^{\prime}$.

Definition $16 \quad A$ family of parameterized behavior strategy profiles, denoted by $\mathcal{P}$, is a family of functions $\boldsymbol{\beta} \in \mathcal{P}$ such that

$$
\boldsymbol{\beta}:(0,1) \rightarrow B
$$

maps any number $\varepsilon \in(0,1)$ to a behavior strategy profile $\boldsymbol{\beta}(\varepsilon) \in B$. Moreover, we say the following:

- $\boldsymbol{\beta} \in \mathcal{P}$ is completely mixed if $\boldsymbol{\beta}(\varepsilon)$ is always completely mixed;

- $\boldsymbol{\beta} \in \mathcal{P}$ is rational (respectively polynomial) if each probability $\boldsymbol{\beta}_{i}(\varepsilon, c)$ can be expressed as a rational (respectively polynomial) function of $\varepsilon$.

We are now ready to provide a definition of quasi-perfect equilibria more convenient to our work, following Van Damme [1984]. ${ }^{12}$

First, we introduce the idea of best responses at information sets. ${ }^{13}$

\footnotetext{
${ }^{12}$ See also Govindan and Wilson [2012] Definition 4.1.

${ }^{13}$ In the terminology of Van Damme [1984], $u$-best responses are quasi-best replies.
} 
DEFINITION 17 Given a game in extensive form $\Gamma$, a completely mixed behavior strategy profile $\beta \in B$, a player $i \in N$, and an information set $u \in U_{i}$, a behavior strategy $\beta_{i} \in B_{i}$ is an u-best response to $\beta \in B$, written $\beta_{i} \in \mathcal{B R}_{u}(\beta)$, if the following holds:

$$
\max _{\beta_{i}^{\prime} \in B_{i}: \beta_{i}=\beta_{u}^{\prime}} H_{i}\left(\beta /{ }_{u} \beta_{i}^{\prime} \mid u\right)=\max _{\beta_{i}^{\prime} \in B_{i}} H_{i}\left(\beta /{ }_{u} \beta_{i}^{\prime} \mid u\right) .
$$

Intuitively, $\beta_{i}$ is an $u$-best response to $\beta$ whenever playing as prescribed by $\beta_{i}$ at information set $u$ is part of some player $i$ 's optimal strategy in the game following $u$, given that $u$ has been reached and the other players behave as prescribed by $\beta$. In other words, $\beta_{i}$ is a best response to $\beta$ whenever player $i$ ends up playing at information set $u$.

The formal definition of quasi-perfect equilibrium follows. ${ }^{14}$

Definition 18 Given a game in extensive form $\Gamma, \beta \in B$ is a quasi-perfect equilibrium of $\Gamma$ if there exists a completely mixed $\boldsymbol{\beta} \in \mathcal{P}$ such that:

1. $\beta$ is a limit point (as $\varepsilon$ goes to zero) of $\boldsymbol{\beta}(\varepsilon)$;

2. for every player $i \in N$ and information set $u \in U_{i}, \beta_{i} \in \mathcal{B R}_{u}(\boldsymbol{\beta}(\varepsilon))$ holds eventually (i.e., for sufficiently small $\varepsilon$ ).

In words, provided that $\varepsilon \in(0,1)$ is sufficiently small, for every player $i$ and information set $u \in U_{i}, \beta_{i}$ prescribes player $i$ to play an optimal strategy against $\boldsymbol{\beta}(\varepsilon)$, given that $u$ has been reached and she will play optimally in the rest of the game (assuming opponents follow strategies in $\boldsymbol{\beta}(\varepsilon)$ ). This condition encodes the fact that a player only considers the possibility of opponents' future trembles, while she does not care about hers.

Note that the game in extensive form of Figure 1 also shows that, in order to span the entire set of quasi perfect equilibria, it is necessary to use nonpolynomial $\boldsymbol{\beta} \in \mathcal{P}$. In particular, in order to get $\beta_{2}(a) \in(0,1)$, we must have $\boldsymbol{\beta}_{1}(D)=\frac{\boldsymbol{\beta}_{1}(B)}{\boldsymbol{\beta}_{1}(A)}$, and this rational restriction is generally required any time in an equilibrium we have to combine sequential rationality and fully mixed behavior strategies. This is formally shown by Theorem 4.1 of Hammond [1994], where he proves that equilibrium refinements have four equivalent characterizations: (i) complete conditional probability systems; ${ }^{15}$ (ii) lexicographic hierarchies of probabilities; ${ }^{16}$ (iii) extended logarithmic likelihood ratios; and (iv) certain "canonical rational probability functions" representing "trembles" directly as infinitesimal probabilities. In particular, Kohlberg and Reny [1997] prove that sequential equilibria can be characterized in terms of relative probabilities that compare the likelihoods of any pair of events, even those with probability zero. ${ }^{17}$ More

\footnotetext{
${ }^{14}$ See Van Damme [1984] Lemma 2.

${ }^{15}$ See Myerson [1986] and McLennan [1989].

${ }^{16}$ See Blume et al. [1991] and Govindan and Klumpp [2003].

${ }^{17}$ Note that quasi perfect equilibria are a refinement of sequential equilibria.
} 
generally, Blume and Zame [1994] prove that the equilibrium correspondences of extensive-form perfect and sequential equilibria are semi-algebraic sets and, therefore, they can be defined by finite systems of polynomial inequalities. For the sake of this paper, we can sum up these results in the following statement: any quasi-perfect equilibrium can be obtained as limit point (as $\varepsilon$ goes to zero) of $\boldsymbol{\beta}(\varepsilon)$ where the behavioral strategy profiles $\boldsymbol{\beta}(\varepsilon) \in B$ can be described using rational functions of $\varepsilon$. This crucial property allows us to restrict our attention to rational functions of $\varepsilon$. Formally:

REMARK 2 Given a game in extensive form $\Gamma$, let $\beta \in B$ be a quasi-perfect equilibrium of $\Gamma$. Then, there exists a completely mixed $\boldsymbol{\beta} \in \mathcal{P}$ such that $\boldsymbol{\beta}$ is rational and conditions (1) and (2) in Definition 18 hold.

\subsection{Perturbed Games in Extensive and in Sequence Form}

Before entering into the details of the proof of our characterization, it is useful to understand the different ways of working of the perturbations when applied to the different forms. Indeed, similar perturbations applied to different game forms produce different refinements. In the following, we discuss the connection between perturbations for the extensive form and those for the sequence form.

As discussed in Section 2.2, given a game in extensive form and its corresponding sequence form, we can transform behavior strategies into realization plans and vice versa, even if the transformation from realization plans to behavior strategies is not uniquely determined. However, things are slightly more complex with perturbed games.

First, let us consider perturbed games in extensive form. Let a Selten perturbed game in extensive form $\Gamma^{S e}=(\Gamma, \eta)$ be given. Since for any $i \in N, u \in U_{i}$, and $c \in C_{u}$

$$
\beta_{i}(c)=\frac{r_{i}(\sigma(u) c)}{r_{i}(\sigma(u))} \geq \eta(c),
$$

we can build an associated perturbed game in sequence form $\left(\Gamma_{\boldsymbol{S}}, \eta\right)$ such that, for any $i \in N, u_{i} \in U_{i}$, and $c \in C_{u}$, it must be the case that:

$$
r_{i}(\sigma(u) c) \geq \eta(c) r_{i}(\sigma(u)) .
$$

This means that, in the associated perturbed game in sequence form, the realization plan of a sequence $\sigma(u) c$ is subject to a lower bound that depends on the realization plan of the sequence $\sigma(u){ }^{18}$

On the other hand, given a Miltersen-Sørensen perturbed game $\Gamma^{M S}=\left(\Gamma_{\boldsymbol{S}}, \varepsilon\right)$, since for any $i \in N, u \in U_{i}$, and $c^{\prime} \in C_{u}$

$$
r_{i}\left(\sigma(u) c^{\prime}\right)=\prod_{c \in \sigma(u)} \beta_{i}(c) \beta_{i}\left(c^{\prime}\right) \geq \varepsilon^{\left|\sigma(u) c^{\prime}\right|},
$$

\footnotetext{
${ }^{18}$ Farina and Gatti [2017] show how an extensive-form perfect equilibrium can be computed by means of the sequence form.
} 
we can construct an associated perturbed game in extensive form $(\Gamma, \varepsilon)$ where for any $i \in N, u \in U_{i}$, and $c^{\prime} \in C_{u}$ it must hold:

$$
\beta_{i}\left(c^{\prime}\right) \geq \frac{\varepsilon^{\left|\sigma(u) c^{\prime}\right|}}{\prod_{c \in \sigma(u)} \beta_{i}(c)} .
$$

This means that, in the associated perturbed game in extensive form, the behavior strategy of a choice $c^{\prime}$ is subject to a lower bound that depends on the probabilities of the choices $c \in \sigma(u)$. Such a lower bound is not compliant with the definition of the Selten perturbed game, which instead requires that the lower bound depends only on $\varepsilon$ and not on $\beta_{i}$.

\section{NASH EQUILIBRIA OF PERTURBED GAMES}

In this section, we introduce a class of $n$-player perturbed games in sequence form and prove some properties of their Nash equilibria, which turn out to be useful in proving the core results in Sections 5 and 6.

\subsection{A General Approach to Perturbation in Sequence Form}

Next, we introduce our class of $n$-player perturbed games in sequence form, which generalizes those studied by Miltersen and Sørensen [2010].

DeFINITION 19 Given a game in sequence form $\Gamma_{\boldsymbol{S}}$ and a player $i \in N$, let $\ell_{i}:(0,1) \times S_{i} \mapsto \mathbb{R}$ be a function that maps any number $\varepsilon \in(0,1)$ and sequence $s_{i} \in S_{i}$ to a lower bound $\ell_{i}\left(\varepsilon, s_{i}\right)$ such that:

1. $\ell_{i}\left(\varepsilon, s_{i}\right)$ is a polynomial in $\varepsilon$, for every $s_{i} \in S_{i}$;

2. $\lim _{\varepsilon \rightarrow 0^{+}} \ell_{i}\left(\varepsilon, s_{i}\right)=0$, for every $s_{i} \in S_{i} \backslash\{\varnothing\}$, while $\ell_{i}(\varepsilon, \varnothing)=1$;

3. $\lim _{\varepsilon \rightarrow 0^{+}} \frac{\ell_{i}\left(\varepsilon, s_{i} \mid c\right)}{\ell_{i}\left(\varepsilon, s_{i}\right)}=0$, for every $s_{i} \in S_{i}$ and $c \in C_{u}$ such that $s_{i} \mid c \in S_{i}$.

Then, we define an $\ell_{i}$-quasi-perfect perturbation for $R_{i}$ as a function $\varepsilon \mapsto$ $R_{i}(\varepsilon)$ defined over $\varepsilon \in(0,1)$ in which $R_{i}(\varepsilon)$ is the set of all the realization plans $r_{i} \in R_{i}$ such that $r_{i}\left(s_{i}\right) \geq \ell_{i}\left(\varepsilon, s_{i}\right)$ for every $s_{i} \in S_{i}$.

In words, an $\ell_{i}$-quasi-perfect perturbation constrains player $i$ to play every sequence $s_{i} \in S_{i}$ with probability larger than or equal to the lower-bound $\ell_{i}\left(\varepsilon, s_{i}\right)$. Furthermore, the lower-bounds enjoy particular properties:

1. they can be expressed as polynomials in $\varepsilon$;

2. they approach zero as $\varepsilon$ goes to zero;

3. for every sequence $s_{i} \in S_{i}$ and choice $c \in C_{u}$ such that $s_{i} \mid c \in S_{i}, \ell_{i}\left(\varepsilon, s_{i} \mid c\right)$ approaches zero faster than $\ell_{i}\left(\varepsilon, s_{i}\right)$.

DEFINITION 20 Given a game in extensive form $\Gamma$, we call perturbed sequence form of $\Gamma$ the sequence form $\Gamma_{\boldsymbol{S}}$ of $\Gamma$ together with an $\ell_{i}$-quasi-perfect perturbation for $R_{i}$, for every player $i \in N$. 
In the following, the tuple $\left(\Gamma_{S},\left\{\ell_{i}\right\}_{i \in N}\right)$ denotes a perturbed sequence form of $\Gamma$, defined for some $\ell_{i}$-quasi-perfect perturbations. Moreover, we let $\Gamma_{\boldsymbol{S}}(\varepsilon)$ be a particular perturbed game in sequence form, which is obtained from $\Gamma_{S}$ by restricting each set of realization plans $R_{i}$ to be $R_{i}(\varepsilon) .{ }^{19}$

In matrix notation, we can write the constraints defining $R_{i}(\varepsilon)$ as $r_{i} \geq \ell_{i}(\varepsilon)$ for all $i \in N$, where, overloading notation, $\ell_{i}(\varepsilon)$ is a vector-valued function of $\varepsilon$, defined over $\varepsilon \in(0,1)$, whose components are the functions $\ell_{i}\left(\varepsilon, s_{i}\right)$ for $s_{i} \in S_{i}$. In the following, we use $r(\varepsilon)$ to denote a valid realization plan profile for a perturbed game in sequence form $\Gamma_{\boldsymbol{S}}(\varepsilon)$, i.e., $r_{i}(\varepsilon) \in R_{i}(\varepsilon)$ for all $i \in N$.

\subsection{Properties of Nash Equilibria of Perturbed Games}

The main result of this section is Theorem 1, which provides a characterization of Nash equilibria perturbed games in sequence form, a necessary step to derive that any limit point of a sequence of Nash equilibria of perturbed games in sequence form is a quasi-perfect equilibrium of the original, unperturbed game in extensive form, our generalization of Miltersen and Sørensen [2010] result. To prove Theorem 1, we need to derive some properties that characterize the Nash equilibria of perturbed games in sequence form $\Gamma_{\boldsymbol{S}}(\varepsilon)$. In particular, Lemma 1 is used to prove Lemma 2 that in turn is used to prove Lemma 3, which allows us to prove Theorem 1.

First, we introduce the definition of player $i$ 's expected utility in the sequence form, given that information set $u$ has been reached: given a player $i \in N$ and an information set $u \in U_{i}$, we let

$$
G_{i}(u, r)=\sum_{s \in S: \sigma(u) \sqsubset s_{i}} g_{i}(s) \prod_{j \in N} r_{j}\left(s_{j}\right)
$$

be player $i$ 's expected utility contribution due to terminal nodes that are reachable from $u$, given that realization plan profile $r \in R$ is played.

LEMma 1 Given a perturbed sequence form $\left(\Gamma_{\boldsymbol{S}},\left\{\ell_{i}\right\}_{i \in N}\right), r(\varepsilon)$ is a Nash equilibrium of a perturbed game in sequence form $\Gamma_{\boldsymbol{S}}(\varepsilon)$ if and only if, for every player $i \in N, \tilde{r}_{i}(\varepsilon)=r_{i}(\varepsilon)-\ell_{i}(\varepsilon)$ is an optimal solution to Problem (4).

$$
\begin{array}{ll}
\text { (4a) } \quad \max & \tilde{r}_{i}^{T}\left(M_{i} \prod_{j \neq i \in N} r_{j}(\varepsilon)\right) \\
\text { (4b) } & F_{i} \tilde{r}_{i}=f_{i}-F_{i} \ell_{i}(\varepsilon) \\
\text { (4c) } & \tilde{r}_{i} \geq 0 .
\end{array}
$$

\footnotetext{
${ }^{19}$ Let us remark that $\Gamma_{\boldsymbol{S}}(\varepsilon)$ may not be well-defined for all values of $\varepsilon \in(0,1)$, since some sets $R_{i}(\varepsilon)$ might be empty. Nevertheless, conditions (1)-(3) in Definition 19 ensure that there exists $\bar{\varepsilon} \in(0,1)$ such that $\Gamma_{S}(\varepsilon)$ is well-defined for all $\varepsilon \in(0, \bar{\varepsilon})$. Thus, whenever we refer to a perturbed game in sequence form $\Gamma_{S}(\varepsilon)$, we can safely assume that it is well-defined, provided that $\varepsilon$ is sufficiently small.
} 
Proof: Recall that in a Nash equilibrium every player must play a best response to the opponents' strategies. Let us fix a player $i \in N$. Consider the best-response problem of player $i$ against $r(\varepsilon)$ in $\Gamma_{\boldsymbol{S}}(\varepsilon)$, then introducing variables $\tilde{r}_{i}=r_{i}-\ell_{i}(\varepsilon)$, the problem can be rewritten as follows:

$$
\begin{aligned}
\max & \tilde{r}_{i}^{T}\left(M_{i} \prod_{j \neq i \in N} r_{j}(\varepsilon)\right)+\ell_{i}(\varepsilon)^{T}\left(M_{i} \prod_{j \neq i \in N} r_{j}(\varepsilon)\right) \quad \text { s.t. } \\
& F_{i} \tilde{r}_{i}=f_{i}-F_{i} \ell_{i}(\varepsilon) \\
& \tilde{r}_{i} \geq 0
\end{aligned}
$$

which is equivalent to Problem (4) since the second term in the objective function is a constant.

Q.E.D.

Let us remark that Problem (4) is a linear program, as all players' realization plans are fixed, except for that of player $i$. In the following remark, we introduce the dual of Problem (4).

REMARK 3 Given a perturbed sequence form $\left(\Gamma_{\boldsymbol{S}},\left\{\ell_{i}\right\}_{i \in N}\right)$, for every realization plan profile $r(\varepsilon)$ of $\Gamma_{\boldsymbol{S}}(\varepsilon)$ and player $i \in N$, Problem (5) is the dual of Problem (4), where $v \in \mathbb{R}^{\left|U_{i}\right|+1}$ denotes the vector of dual variables associated with Constraints (4a):

$$
\begin{array}{ll}
\min & \left(f_{i}-F_{i} \ell_{i}(\varepsilon)\right)^{T} v \\
& F_{i}^{T} v \geq\left(M_{i} \prod_{j \neq i \in N} r_{j}(\varepsilon)\right)
\end{array}
$$

The following remark introduces an equivalent way of expressing the constraints in Problem (5), where the conditions that each variable $v_{u}$ must satisfy are made explicit.

Remark 4 Constraints (5a) in Problem (5) are equivalent to:

$$
v_{u} \geq \sum_{\substack{s \in S: \\ s_{i}=\sigma(u) c}}\left[g_{i}(s) \prod_{j \neq i \in N} r_{j}\left(\varepsilon, s_{j}\right)\right]+\sum_{\substack{u^{\prime} \in U_{i}: \\ \sigma\left(u^{\prime}\right)=\sigma(u) c}} v_{u^{\prime}},
$$

for every information set $u \in U_{i}$ and choice $c \in C_{u}$.

The optimal solutions to Problem (5) enjoy some particular properties, which are stated in the following lemmas. The first lemma says that, in an optimal solution, each variable $v_{u}$ is set to the value of player $i$ 's expected utility for an optimal strategy in the game following $u$, and this holds for any $u \in U_{i}$ because of perturbations.. 
Lemma 2 Given a perturbed sequence form $\left(\Gamma_{\boldsymbol{S}},\left\{\ell_{i}\right\}_{i \in N}\right)$, for every realization plan profile $r(\varepsilon)$ of $\Gamma_{\boldsymbol{S}}(\varepsilon)$ and player $i \in N, v^{*} \in \mathbb{R}^{\left|U_{i}\right|+1}$ is an optimal solution to Problem (5) if and only if, for every $u \in U_{i}$, it holds:

$$
v_{u}^{*}=\max _{\substack{r_{i}^{\prime} \in R_{i}: \\ r_{i}^{\prime}(\sigma(u))=1}} G_{i}\left(u, r(\varepsilon) / r_{i}^{\prime}\right)
$$

Proof: Let us consider Problem (5). First, observe that the objective function coefficient of the first component of $v$ is always zero, since $\ell_{i}(\varepsilon, \emptyset)=1$ by Definition 19. Moreover, for every information set $u \in U_{i}$, the objective function coefficient for the component $v_{u}$ associated with $u$ is equal to $\ell_{i}(\varepsilon, \sigma(u))-$ $\sum_{c \in \sigma(u)} \ell_{i}(\varepsilon, \sigma(u) c)$. Then, in an optimal solution $v^{*} \in \mathbb{R}^{\left|U_{i}\right|+1}$ to Problem (5), each variable $v_{u}$ is set to its minimum value given Constraints (6).

$(\Longleftarrow)$ Suppose $v^{*} \in \mathbb{R}^{\left|U_{i}\right|+1}$ is an optimal solution to Problem (5). We prove Equation (7) using a simple inductive argument. The base case of the induction is when there is no information set $u^{\prime} \in U_{i}$ with $u \prec u^{\prime}$. For every choice $c \in \sigma(u)$, the following must hold:

$$
v_{u} \geq \sum_{\substack{s \in S: \\ s_{i}=\sigma(u) c}}\left[g_{i}(s) \prod_{j \neq i \in N} r_{j}\left(\varepsilon, s_{j}\right)\right]
$$

which, using the fact that $v_{u}^{*}$ must be set to its minimum possible value given the constraints, implies the following:

$$
v_{u}^{*}=\max _{c \in \sigma(u)} \sum_{\substack{s \in S: \\ s_{i}=\sigma(u) c}}\left[g_{i}(s) \prod_{j \neq i \in N} r_{j}\left(\varepsilon, s_{j}\right)\right]=\max _{\substack{r_{i}^{\prime} \in R_{i}: \\ r_{i}^{\prime}(\sigma(u))=1}} G_{i}\left(u, r(\varepsilon) / r_{i}^{\prime}\right),
$$

where last equality holds since there is no $u^{\prime} \in U_{i}$ such that $u \prec u^{\prime}$ and $\sum_{c \in \sigma(u)} r_{i}^{\prime}(\sigma(u) c)=r_{i}^{\prime}(\sigma(u))=1$, for the definition of realization plan. As for the inductive step, let us consider an information set $u \in U_{i}$ and assume, by induction, that Equation (7) holds for every information set $u^{\prime} \in U_{i}$ with $u \prec u^{\prime}$. 
We can write:

$$
\begin{aligned}
& v_{u}^{*}=\max _{c \in \sigma(u)}\left\{\sum_{\substack{s \in S: \\
s_{i}=\sigma(u) c}}\left[g_{i}(s) \prod_{j \neq i \in N} r_{j}\left(\varepsilon, s_{j}\right)\right]+\sum_{\substack{u^{\prime} \in U_{i}: \\
\sigma\left(u^{\prime}\right)=\sigma(u) c}} v_{u^{\prime}}^{*}\right\}= \\
& =\max _{c \in \sigma(u)}\left\{\sum_{\substack{s \in S: \\
s_{i}=\sigma(u) c}}\left[g_{i}(s) \prod_{j \neq i \in N} r_{j}\left(\varepsilon, s_{j}\right)\right]+\right. \\
& \left.+\sum_{\substack{u^{\prime} \in U_{i}: \\
\sigma\left(u^{\prime}\right)=\sigma(u) c}} \max _{\substack{r_{i}^{\prime} \in R_{i}: \\
r_{i}^{\prime}\left(\sigma\left(u^{\prime}\right)\right)=1}} G_{i}\left(u, r(\varepsilon) / r_{i}^{\prime}\right)\right\}= \\
& =\max _{\substack{r_{i}^{\prime} \in R_{i}: \\
r_{i}^{\prime}(\sigma(u))=1}} G_{i}\left(u, r(\varepsilon) / r_{i}^{\prime}\right),
\end{aligned}
$$

where the first equality directly follows from the optimality of $v^{*}$, the second one from the inductive hypothesis, while the last equality holds since we have $\sum_{c \in \sigma(u)} r_{i}^{\prime}(\sigma(u) c)=r_{i}^{\prime}(\sigma(u))=1$.

$(\Longrightarrow)$ By contradiction, suppose that Equation (7) holds and $v^{*} \in \mathbb{R}^{\left|U_{i}\right|+1}$ is not an optimal solution to Problem (5). Thus, there must be an information set $u \in U_{i}$ such that Equation (7) holds for all $u^{\prime} \in U_{i}$ with $u \prec u^{\prime}$ and $v_{u}^{*}$ satisfies all the Constraints (6) with strict inequality. Thus,

$$
\begin{aligned}
v_{u}^{*}>\max _{c \in \sigma(u)}\left\{\sum_{\substack{s \in S: \\
s_{i}=\sigma(u) c}}\left[g_{i}(s) \prod_{j \neq i \in N} r_{j}\left(\varepsilon, s_{j}\right)\right]+\sum_{\substack{u^{\prime} \in U_{i}: \\
\sigma\left(u^{\prime}\right)=\sigma(u) c}} v_{u^{\prime}}^{*}\right\}= \\
=\max _{\substack{r_{i}^{\prime} \in R_{i}: \\
r_{i}^{\prime}(\sigma(u))=1}} G_{i}\left(u, r(\varepsilon) / r_{i}^{\prime}\right),
\end{aligned}
$$

which contradicts Equation (7) for the information set $u$.

Q.E.D.

The following lemma says that, if an optimal solution to Problem (5) satisfies Constraint (6), for an information set $u$ and a choice $c \in C_{u}$, with equality, then playing choice $c$ at $u$ is part of an optimal strategy in the game following $u$, and this holds for any $u \in U_{i}$ because of perturbations.

Lemma 3 Given a perturbed sequence form $\left(\Gamma_{\boldsymbol{S}},\left\{\ell_{i}\right\}_{i \in N}\right)$, for every realization plan profile $r(\varepsilon)$ of $\Gamma_{S}(\varepsilon)$, player $i \in N$, information set $u \in U_{i}$, and choice 
$c \in C_{u}$, Constraint (6) holds with equality in an optimal solution to Problem (5) if and only if:

$$
\max _{\substack{r_{i}^{\prime} \in R_{i}: \\ r_{i}^{\prime}(\sigma(u) c)=1}} G_{i}\left(u, r(\varepsilon) / r_{i}^{\prime}\right)=\max _{\substack{r_{i}^{\prime} \in R_{i}: \\ r_{i}^{\prime}(\sigma(u))=1}} G_{i}\left(u, r(\varepsilon) / r_{i}^{\prime}\right) .
$$

Proof: In the following, let $v^{*} \in \mathbb{R}^{\left|U_{i}\right|+1}$ be optimal for Problem (5).

$(\Longrightarrow)$ Using Lemma 2 and Equation (8), we can write:

$$
\begin{aligned}
v_{u}^{*} & =\max _{\substack{r_{i}^{\prime} \in R_{i}: \\
r_{i}^{\prime}(\sigma(u))=1}} G_{i}\left(u, r(\varepsilon) / r_{i}^{\prime}\right)=\max _{\substack{r_{i}^{\prime} \in R_{i} \\
r_{i}^{\prime}(\sigma(u) c)=1}} G_{i}\left(u, r(\varepsilon) / r_{i}^{\prime}\right)= \\
& =\sum_{\substack{s \in S: \\
s_{i}=\sigma(u) c}}\left[g_{i}(s) \prod_{j \neq i \in N} r_{j}\left(\varepsilon, s_{j}\right)\right]+\sum_{\substack{u^{\prime} \in U_{i}: \\
\sigma\left(u^{\prime}\right)=\sigma(u) c}} v_{u^{\prime}}^{*} .
\end{aligned}
$$

$(\Longleftarrow)$ Using Lemma 2 we can write:

$$
\begin{aligned}
v_{u}^{*} & =\sum_{\substack{s \in S: \\
s_{i}=\sigma(u) c}}\left[g_{i}(s) \prod_{j \neq i \in N} r_{j}\left(\varepsilon, s_{j}\right)\right]+\sum_{\substack{u^{\prime} \in U_{i}: \\
\sigma\left(u^{\prime}\right)=\sigma(u) c}} v_{u^{\prime}}^{*}= \\
& =\max _{\substack{r_{i}^{\prime} \in R_{i} \\
r_{i}^{\prime}(\sigma(u) c)=1}} G_{i}\left(u, r(\varepsilon) / r_{i}^{\prime}\right)=\max _{\substack{r_{i}^{\prime} \in R_{i}: \\
r_{i}^{\prime}(\sigma(u))=1}} G_{i}\left(u, r(\varepsilon) / r_{i}^{\prime}\right) .
\end{aligned}
$$

This concludes the proof.

Q.E.D.

Now, we are ready to prove a fundamental property of the Nash equilibria of perturbed games in sequence form $\Gamma_{\boldsymbol{S}}(\varepsilon)$.

THEOREM 1 Given a perturbed sequence form $\left(\Gamma_{\boldsymbol{S}},\left\{\ell_{i}\right\}_{i \in N}\right)$, a realization plan profile $r(\varepsilon)$ is a Nash equilibrium of $\Gamma_{\boldsymbol{S}}(\varepsilon)$ if and only if, for every player $i \in N$, information set $u \in U_{i}$, and choice $c \in C_{u}$, it holds that:

1. if $r_{i}(\varepsilon, \sigma(u) c)>\ell_{i}(\varepsilon, \sigma(u) c)$, then

$$
\max _{\substack{r_{i}^{\prime} \in R_{i}: \\ r_{i}^{\prime}(\sigma(u) c)=1}} G_{i}\left(u, r(\varepsilon) / r_{i}^{\prime}\right)=\max _{\substack{r_{i}^{\prime} \in R_{i}: \\ r_{i}^{\prime}(\sigma(u))=1}} G_{i}\left(u, r(\varepsilon) / r_{i}^{\prime}\right)
$$

2. if $\max _{\substack{r_{i}^{\prime} \in R_{i}: \\ r_{i}^{\prime}(\sigma(u) c)=1}} G_{i}\left(u, r(\varepsilon) / r_{i}^{\prime}\right)<\max _{\substack{r_{i}^{\prime} \in R_{i}: \\ r_{i}^{\prime}(\sigma(u))=1}} G_{i}\left(u, r(\varepsilon) / r_{i}^{\prime}\right)$, then

$$
r_{i}(\varepsilon, \sigma(u) c)=\ell_{i}(\varepsilon, \sigma(u) c) .
$$


Proof: Recall that, by Lemma 1, $r(\varepsilon)$ is a Nash equilibrium of $\Gamma_{\boldsymbol{S}}(\varepsilon)$ if and only if, for every player $i \in N, \tilde{r}_{i}(\varepsilon)=r_{i}(\varepsilon)-\ell_{i}(\varepsilon)$ is an optimal solution to Problem (4). The result is a direct application of the complementarity slackness theorem in linear programming. Let us fix $i \in N, u \in U_{i}$, and $c \in \sigma(u)$. By applying the theorem to Problems (4) and (5) we can conclude that $\tilde{r}_{i}(\varepsilon)$ and $v^{*} \in \mathbb{R}^{\left|U_{i}\right|+1}$ are optimal if and only if the following holds:

1. if $\tilde{r}_{i}(\varepsilon, \sigma(u) c)>0$ (i.e., equivalently, $r_{i}(\varepsilon, \sigma(u) c)>\ell_{i}(\varepsilon, \sigma(u) c)$ ), then Constraint (6) for information set $u$ and choice $c$ must hold with equality, which, using Lemma 3, implies Equation (8);

2. if Constraint (6), for information set $u$ and choice $c$, does not hold with equality, then it must be $\tilde{r}_{i}(\varepsilon, \sigma(u) c)=0$ (i.e., equivalently, $r_{i}(\varepsilon, \sigma(u) c)=$ $\left.\ell_{i}(\varepsilon, \sigma(u) c)\right)$. Notice that the constraint holds with strict inequality whenever

$$
\max _{\substack{r_{i}^{\prime} \in R_{i}: \\ r_{i}^{\prime}(\sigma(u) c)=1}} G_{i}\left(u, r(\varepsilon) / r_{i}^{\prime}\right)<\max _{\substack{r_{i}^{\prime} \in R_{i}: \\ r_{i}^{\prime}(\sigma(u))=1}} G_{i}\left(u, r(\varepsilon) / r_{i}^{\prime}\right),
$$

by Lemma 3 .

Since the two conditions hold for every player $i \in N$, information set $u \in U_{i}$, and choice $c \in \sigma(u)$, the proof is complete.

Q.E.D.

Intuitively, in a Nash equilibrium of $\Gamma_{\boldsymbol{S}}(\varepsilon)$, player $i$ plays sequence $\sigma(u) c$ with probability strictly greater than its lower-bound $\ell_{i}(\varepsilon, \sigma(u) c)$ only if playing $c$ is part of some optimal strategy in the game following $u$. Conversely, whenever $c \in C_{u}$ is not part of any optimal strategy in the game following $u$, it must be the case that sequence $\sigma(u) c$ is played with minimum probability. Theorem 1 formally expresses the idea that, in a perturbed game in sequence form $\Gamma_{\boldsymbol{S}}(\varepsilon)$, when a player decides how to play in a given information set, she does not take into account her future trembles, but only opponents' ones.

\section{NASH EQUILIBRIA OF PERTURBED GAMES IN SEQUENCE FORM ARE QUASI-PERFECT EQUILIBRIA}

In this section, we prove the first part of our main result: all limit points of sequences of Nash equilibria of $n$-player perturbed games $\Gamma_{S}(\varepsilon)$ are quasi-perfect equilibria of the game in extensive form $\Gamma$. Note that this result is a generalization of Lemma 1 by Miltersen and Sørensen [2010], however, our proof is significantly different, since there is no a direct way to extend their proof to the general case of our perturbed games.

First, we provide the following definition.

DEFINITIOn $21 \quad A$ family of parameterized realization plan profiles, denoted by $\mathcal{R}$, is a family of functions $\boldsymbol{r} \in \mathcal{R}$ such that

$$
\boldsymbol{r}:(0,1) \mapsto R
$$


maps any number $\varepsilon \in(0,1)$ to a realization plan profile $\boldsymbol{r}(\varepsilon) \in R$. Moreover, we say the following:

- $\boldsymbol{r} \in \mathcal{R}$ is completely mixed if $\boldsymbol{r}(\varepsilon)$ is always completely mixed;

- $\boldsymbol{r} \in \mathcal{R}$ is rational (respectively polynomial) if each term $\boldsymbol{r}_{i}\left(\varepsilon, s_{i}\right)$ can be expressed as a rational (respectively polynomial) function of $\varepsilon$.

Then, we provide a characterization of $u$-best responses in terms of the sequence form.

Lemma 4 Given a game in extensive form $\Gamma$, a completely mixed behavior strategy profile $\beta \in B$, a player $i \in N$, and an information set $u \in U_{i}$, a behavior strategy $\beta_{i} \in B_{i}$ is a u-best response to $\beta \in B$, i.e., $\beta_{i} \in \mathcal{B R}_{u}(\beta)$, if and only if the following holds for every choice $c \in C_{u}$ :

$$
\beta_{i}(c)>0 \Longrightarrow \max _{\substack{r_{i}^{\prime} \in R_{i}: \\ r_{i}^{\prime}(\sigma(u) c)=1}} G_{i}\left(u, r / r_{i}^{\prime}\right)=\max _{\substack{r_{i}^{\prime} \in R_{i}: \\ r_{i}^{\prime}(\sigma(u))=1}} G_{i}\left(u, r / r_{i}^{\prime}\right),
$$

where $r \in R$ is the realization plan of $\beta$.

Proof: First, let us notice that the following relation holds:

$$
\begin{aligned}
\max _{\substack{r_{i}^{\prime} \in R_{i}: \\
r_{i}^{\prime}(\sigma(u) c)=1}} G_{i}\left(u, r / r_{i}^{\prime}\right) & =\max _{\substack{r_{i}^{\prime} \in R_{i}: \\
r_{i}^{\prime}(\sigma(u))=1}} G_{i}\left(u, r / r_{i}^{\prime}\right) \Longleftrightarrow \\
\max _{\beta_{i}^{\prime} \in B_{i}: \beta_{i}^{\prime}(c)=1} H_{i}\left(\beta /{ }_{u} \beta_{i}^{\prime} \mid u\right) & =\max _{\beta_{i}^{\prime} \in B_{i}} H_{i}\left(\beta /{ }_{u} \beta_{i}^{\prime} \mid u\right) .
\end{aligned}
$$

In order to see this, for $z \in Z(u)$ and $\beta_{i}^{\prime} \in B_{i}$, let $H_{i}\left(z, \beta, \beta_{i}^{\prime}\right)$ be such that:

$$
H_{i}\left(z, \beta, \beta_{i}^{\prime}\right)=h_{i}(z)\left(\prod_{j \neq i \in N} \prod_{c \in p(z) \cap D_{j}} \beta_{j}(c)\right)\left(\prod_{c \in p(z) \cap D_{i}: c \notin \sigma(u)} \beta_{i}^{\prime}(c)\right) .
$$

Since $r$ is the realization plan of $\beta$ and $r_{i}^{\prime}(\sigma(u))=1$, the first line of Equation (9) is the same as:

$$
\max _{\beta_{i}^{\prime} \in B_{i}: \beta_{i}^{\prime}(c)=1} \sum_{z \in Z(u)} H_{i}\left(z, \beta, \beta_{i}^{\prime}\right)=\max _{\beta_{i}^{\prime} \in B_{i}} \sum_{z \in Z(u)} H_{i}\left(z, \beta, \beta_{i}^{\prime}\right) .
$$

Then, by dividing both sides of the equality by $\sum_{x \in u} \prod_{j \neq i \in N} \prod_{c \in p(x) \cap D_{j}} \beta_{i}(c)$ and using the definition of $H_{i}(\beta \mid u)$, i.e.,

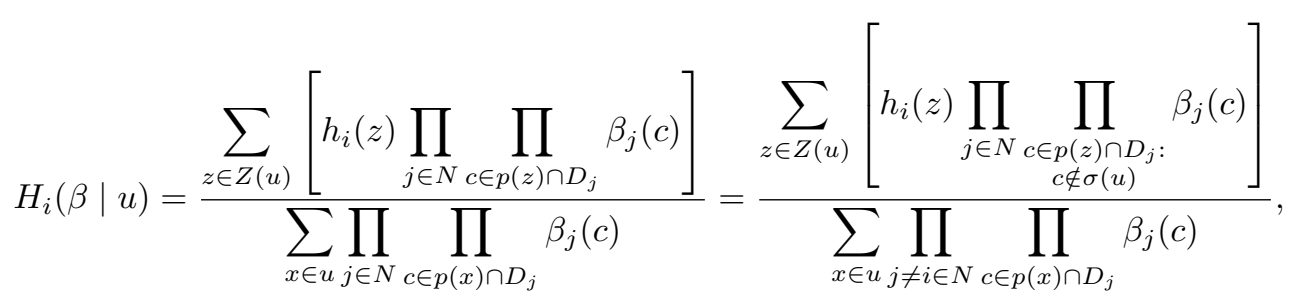


we get the second line of Equation (9). Clearly, we can prove the other direction of Equation (9) following the same steps in the reverse order (and multiplying instead of dividing).

$(\Longrightarrow)$ Suppose that the condition holds for every choice $c \in C_{u}$. Clearly,

$$
\max _{\beta_{i}^{\prime} \in B_{i}: \beta_{i}=\beta_{u}^{\prime}} H_{i}\left(\beta /{ }_{u} \beta_{i}^{\prime} \mid u\right)=\sum_{c \in C_{u}} \beta_{i}(c) \max _{\beta_{i}^{\prime} \in B_{i}: \beta_{i}^{\prime}(c)=1} H_{i}\left(\beta /{ }_{u} \beta_{i}^{\prime} \mid u\right),
$$

and, since $\beta_{i}(c)>0$ only if $\max _{\substack{r_{i}^{\prime} \in R_{i} \\ r_{i}^{\prime}(\sigma(u) c)=1}} G_{i}\left(u, r / r_{i}^{\prime}\right)=\max _{\substack{r_{i}^{\prime} \in R_{i}: \\ r_{i}^{\prime}(\sigma(u))=1}} G_{i}\left(u, r / r_{i}^{\prime}\right)$, a direct application of Equation (9) proves the result.

$(\Longleftarrow)$ As for the other direction, let $\beta_{i} \in \mathcal{B R}_{u}(\beta)$ be given. Thus, we have $\max _{\beta_{i}^{\prime} \in B_{i}: \beta_{i}=\beta_{u}^{\prime}} H_{i}\left(\beta /{ }_{u} \beta_{i}^{\prime} \mid u\right)=\max _{\beta_{i}^{\prime} \in B_{i}} H_{i}\left(\beta /{ }_{u} \beta_{i}^{\prime} \mid u\right)$. By contradiction, suppose that

$\max _{\substack{r_{i}^{\prime} \in R_{i} \\ r_{i}^{\prime}(\sigma(u) c)=1}} G_{i}\left(u, r / r_{i}^{\prime}\right)<\max _{\substack{r_{i}^{\prime} \in R_{i}: \\ r_{i}^{\prime}(\sigma(u))=1}} G_{i}\left(u, r / r_{i}^{\prime}\right)$ for some choice $c \in \sigma(u)$ such that $\beta_{i}(c)>0$. By Equation (9), we would have

$$
\max _{\beta_{i}^{\prime} \in B_{i}: \beta_{i}^{\prime}(c)=1} H_{i}\left(\beta /{ }_{u} \beta_{i}^{\prime} \mid u\right)<\max _{\beta_{i}^{\prime} \in B_{i}} H_{i}\left(\beta /{ }_{u} \beta_{i}^{\prime} \mid u\right),
$$

which implies the following

$$
\begin{aligned}
\max _{\beta_{i}^{\prime} \in B_{i}: \beta_{i}=\beta_{u}^{\prime}} H_{i}\left(\beta /{ }_{u} \beta_{i}^{\prime} \mid u\right) & =\sum_{c \in C_{u}} \beta_{i}(c) \max _{\beta_{i}^{\prime} \in B_{i}: \beta_{i}^{\prime}(c)=1} u_{i}\left(\beta /{ }_{u} \beta_{i}^{\prime} \mid u\right) \\
& <\max _{\beta_{i}^{\prime} \in B_{i}} u_{i}\left(\beta /{ }_{u} \beta_{i}^{\prime} \mid u\right),
\end{aligned}
$$

which contradicts the fact that $\beta_{i} \in \mathcal{B R}_{u}(\beta)$.

Q.E.D.

Intuitively, a behavior strategy $\beta_{i}$ is a $u$-best response to $\beta$ if and only if it places positive probability only on choices $c \in C_{u}$ that are part of some player $i$ 's optimal strategy in the game following information set $u$.

THEOREM 2 Given a perturbed sequence form $\left(\Gamma_{\boldsymbol{S}},\left\{\ell_{i}\right\}_{i \in N}\right)$, let:

1. $\boldsymbol{r} \in \mathcal{R}$ be such that $\boldsymbol{r}(\varepsilon)$ is a Nash equilibrium of $\Gamma_{\boldsymbol{S}}(\varepsilon)$;

2. $\boldsymbol{\beta} \in \mathcal{P}$ be such that $\boldsymbol{\beta}(\varepsilon)$ has $\boldsymbol{r}(\varepsilon)$ as realization plan.

Then, any limit point (as $\varepsilon$ goes to zero) of $\boldsymbol{\beta}(\varepsilon)$ is a quasi-perfect equilibrium of $\Gamma$.

Proof: Let us fix $\boldsymbol{r} \in \mathcal{R}$ such that $\boldsymbol{r}(\varepsilon)$ is a Nash equilibrium of $\Gamma_{\boldsymbol{S}}(\varepsilon)$, and let $\beta \in B$ be a limit point (as $\varepsilon$ goes to zero) of $\boldsymbol{\beta}(\varepsilon)$. In order to prove that $\beta$ is a quasi-perfect equilibrium of $\Gamma$, it is sufficient to show that condition (2) in Definition 18 holds. First, notice that there must exist $\bar{\varepsilon} \in(0,1)$ such that, for all $\varepsilon \in(0, \bar{\varepsilon})$, and for every player $i \in N$, information set $u \in U_{i}$, and choice $c \in \sigma(u)$, if $\beta_{i}(c)>0$, then $\boldsymbol{r}_{i}(\varepsilon, \sigma(u) c)>\ell_{i}(\varepsilon, \sigma(u) c)$. Otherwise, by conditions 
(2)-(3) in Definition 19, it would be $\beta_{i}(c)=0$. In the following, let us fix $i \in N$, $u \in U_{i}$, and $c \in \sigma(u)$. Suppose that $\beta_{i}(c)>0$. For all $\varepsilon \in(0, \bar{\varepsilon})$, we have that $\boldsymbol{r}_{i}(\varepsilon, \sigma(u) c)>\ell_{i}(\varepsilon, \sigma(u) c)$, which, by Theorem 1, implies the following:

$$
\max _{\substack{r_{i}^{\prime} \in R_{i} \\ r_{i}^{\prime}(\sigma(u) c)=1}} G_{i}\left(u, \boldsymbol{r}(\varepsilon) / r_{i}^{\prime}\right)=\max _{\substack{r_{i}^{\prime} \in R_{i}: \\ r_{i}^{\prime}(\sigma(u))=1}} G_{i}\left(u, \boldsymbol{r}(\varepsilon) / r_{i}^{\prime}\right) .
$$

Thus, Lemma 4 allows us to conclude that $\beta_{i} \in \mathcal{B R}_{u}(\boldsymbol{\beta}(\varepsilon))$ eventually, i.e., for $\varepsilon \in(0, \bar{\varepsilon})$. Thus, $\beta \in B$ is a quasi-perfect equilibrium of $\Gamma$.

Q.E.D.

\section{QUASI-PERFECT EQUILIBRIA ARE NASH EQUILIBRIA OF PERTURBED GAMES IN SEQUENCE FORM}

In this section, we prove the second part of our main result: any quasi-perfect equilibrium of a two-player game in extensive form with nature $\Gamma$ can be obtained as limit point of a sequence of Nash equilibria of perturbed games in sequence form $\Gamma_{\boldsymbol{S}}(\varepsilon)$, for a suitably defined perturbed sequence form of $\Gamma$. The general idea of the proof is the following.

1. From Remark 2, we know that any quasi-perfect equilibrium $\beta \in B$ of a game in extensive form $\Gamma$ can be obtained for some completely mixed $\boldsymbol{\beta} \in \mathcal{P}$ such that $\boldsymbol{\beta}$ is rational.

2. Letting $\boldsymbol{r} \in \mathcal{R}$ be such that $\boldsymbol{r}(\varepsilon)$ is the realization plan of $\boldsymbol{\beta}(\varepsilon)$, we apply two transformations to $\boldsymbol{r}$, in order to obtain $\boldsymbol{r}^{*} \in \mathcal{R}$ such that:

- $\boldsymbol{r}^{*}$ is polynomial (instead of being rational, like $\boldsymbol{r}$ );

- for every player $i \in N$ and information set $u \in U_{i}$, player $i$ 's optimal strategies in the game following information set $u$ are preserved when replacing realization plan profile $\boldsymbol{r}(\varepsilon)$ with $\boldsymbol{r}^{*}(\varepsilon)$;

- $\beta$ is a limit point (as $\varepsilon$ goes to zero) of $\boldsymbol{\beta}^{*}(\varepsilon)$, where $\boldsymbol{\beta}^{*} \in \mathcal{P}$ is such that $\boldsymbol{r}^{*}(\varepsilon)$ is the realization plan of $\boldsymbol{\beta}^{*}(\varepsilon)$.

- we can build a particular perturbed sequence form $\left(\Gamma_{\boldsymbol{S}},\left\{\ell_{i}^{\beta}\right\}_{i \in N}\right)$ of $\Gamma$, which depends on $\beta$, such that each $\boldsymbol{r}^{*}(\varepsilon)$ is a valid realization plan for the perturbed game $\Gamma_{\boldsymbol{S}}(\varepsilon)$.

3 . Finally, we prove that $\boldsymbol{r}^{*}(\varepsilon)$ defines a sequence of Nash equilibria of perturbed games $\Gamma_{\boldsymbol{S}}(\varepsilon)$.

We remark that all the results in this section hold for $n$-player games, except for Lemma 5 that can be proved only for two-player games with nature.

\subsection{Transformations}

First, we introduce and analyze two types of transformations that are defined on functions $\boldsymbol{r} \in \mathcal{R}$. The first one allows us to make polynomial a rational $\boldsymbol{r}$. The second kind of transformation applies to a polynomial $\boldsymbol{r} \in \mathcal{R}$ and allows us 
to change the degrees of $\varepsilon$ terms in the polynomials defining $\boldsymbol{r}$. This last step is fundamental for being able to build the perturbed sequence form of interest. In both cases, we show that applying a transformation preserves players' optimal strategies at information sets. ${ }^{20}$

Let us start introducing some useful remarks.

REMARK 5 Given a rational $\boldsymbol{r} \in \mathcal{R}$, for every player $i \in N$ and sequence $s_{i} \in S_{i}$, we can write:

$\boldsymbol{r}_{i}\left(\varepsilon, s_{i}\right)=a_{0}\left(s_{i}\right)+\frac{a_{1}\left(s_{i}\right) \varepsilon+\ldots+a_{d_{N}\left(s_{i}\right)}\left(s_{i}\right) \varepsilon^{d_{N}\left(s_{i}\right)}}{1+b_{1}\left(s_{i}\right) \varepsilon+\ldots+b_{d_{D}\left(s_{i}\right)}\left(s_{i}\right) \varepsilon^{d_{D}\left(s_{i}\right)}}=a_{0}\left(s_{i}\right)+\frac{\operatorname{Poly}_{i}^{N}\left(\varepsilon, s_{i}\right)}{\operatorname{Poly}_{i}^{D}\left(\varepsilon, s_{i}\right)}$,

where $a_{0}\left(s_{i}\right) \in[0,1], a_{x}\left(s_{i}\right) \in \mathbb{R}$ for all $x \in\left\{1, \ldots, d_{N}\left(s_{i}\right)\right\}$, and $b_{x}\left(s_{i}\right) \in \mathbb{R}$ for all $x \in\left\{1, \ldots, d_{D}\left(s_{i}\right)\right\}$, with $d_{N}\left(s_{i}\right), d_{D}\left(s_{i}\right) \in \mathbb{N}$ denoting the degrees of polynomials Poly $_{i}^{N}\left(\varepsilon, s_{i}\right)$ and Poly $_{i}^{D}\left(\varepsilon, s_{i}\right)$, respectively.

REMARK 6 Given a polynomial $\boldsymbol{r} \in \mathcal{R}$, for every player $i \in N$ and sequence $s_{i} \in S_{i}$, we can write:

$$
\boldsymbol{r}_{i}\left(\varepsilon, s_{i}\right)=a_{0}\left(s_{i}\right)+a_{1}\left(s_{i}\right) \varepsilon+\ldots+a_{d\left(s_{i}\right)}\left(s_{i}\right) \varepsilon^{d\left(s_{i}\right)}=\operatorname{Poly}_{i}\left(\varepsilon, s_{i}\right),
$$

where $a_{0}\left(s_{i}\right) \in[0,1]$ and $a_{x}\left(s_{i}\right) \in \mathbb{R}$ for all $1 \in\left\{1, \ldots, d\left(s_{i}\right)\right\}$, with $d\left(s_{i}\right) \in \mathbb{N}$ denoting the degree of the polynomial $\operatorname{Poly}_{i}\left(\varepsilon, s_{i}\right)$.

REMARK 7 Let $\boldsymbol{\beta} \in \mathcal{P}$ be rational. It is easy to prove that $\boldsymbol{r} \in \mathcal{R}$, where $\boldsymbol{r}(\varepsilon)$ is the realization plan of $\boldsymbol{\beta}(\varepsilon)$, is rational too. This is a direct consequence of the fact that the product of rational functions is rational.

Before defining the transformations, let us introduce a motivating example that we will use to explain how they work.

Motivating ExAmPLE Let us consider the game in extensive form of Figure 1, introduced in Section 3.1. As previously discussed, a quasi-perfect equilibrium of the game is the behavior strategy profile $\beta=\left(\beta_{1}, \beta_{2}\right) \in B$ :

$$
\beta_{1}(A)=1, \quad \beta_{1}(C)=1, \quad \text { and } \quad \beta_{2}(a)=\beta_{2}(b)=\frac{1}{2} .
$$

According to Definition $18, \beta$ can be obtained as a limit point (as $\varepsilon$ goes to zero) of $\boldsymbol{\beta}(\varepsilon)$, where $\boldsymbol{\beta} \in \mathcal{P}$ is defined as follows:

$$
\boldsymbol{\beta}_{1}(\varepsilon)=\left[\begin{array}{l}
\boldsymbol{\beta}_{1}(\varepsilon, A)=1-\frac{\varepsilon}{1-\varepsilon} \\
\boldsymbol{\beta}_{1}(\varepsilon, B)=\frac{\varepsilon}{1-\varepsilon} \\
\boldsymbol{\beta}_{1}(\varepsilon, C)=1-\frac{\varepsilon}{1-2 \varepsilon} \\
\boldsymbol{\beta}_{1}(\varepsilon, D)=\frac{\varepsilon}{1-2 \varepsilon}
\end{array}\right] \quad \text { and } \quad \boldsymbol{\beta}_{2}(\varepsilon)=\left[\begin{array}{l}
\boldsymbol{\beta}_{2}(\varepsilon, a)=\frac{1}{2} \\
\boldsymbol{\beta}_{2}(\varepsilon, b)=\frac{1}{2}
\end{array}\right] .
$$

\footnotetext{
${ }^{20}$ This is true in $n$-player games for transformations of the second type, while it only holds in two-player games for transformation of the first type.
} 
Then, we can define $\boldsymbol{r} \in \mathcal{R}$ such that $\mathbf{r}(\varepsilon)$ is the realization plan of $\boldsymbol{\beta}(\varepsilon)$.

$$
\boldsymbol{r}_{1}(\varepsilon)=\left[\begin{array}{l}
\boldsymbol{r}_{1}(\varepsilon, \varnothing)=1 \\
\boldsymbol{r}_{1}(\varepsilon, A)=1-\frac{\varepsilon}{1-\varepsilon} \\
\boldsymbol{r}_{1}(\varepsilon, B)=\frac{\varepsilon}{1-\varepsilon} \\
\boldsymbol{r}_{1}(\varepsilon, A C)=1-\frac{2 \varepsilon}{1-\varepsilon} \\
\boldsymbol{r}_{1}(\varepsilon, A D)=\frac{\varepsilon}{1-\varepsilon}
\end{array}\right] \quad \text { and } \quad \boldsymbol{r}_{2}(\varepsilon)=\left[\begin{array}{l}
\varnothing=1 \\
\boldsymbol{r}_{2}(\varepsilon, a)=\frac{1}{2} \\
\boldsymbol{r}_{2}(\varepsilon, b)=\frac{1}{2}
\end{array}\right] .
$$

Note that, being $\boldsymbol{\beta}$ rational, $\boldsymbol{r}$ is rational too.

Next, we introduce the first type of transformation.

DeFINITION 22 Given a rational $\boldsymbol{r} \in \mathcal{R}$ and a rational function $\varepsilon \mapsto C(\varepsilon)$ defined over $\varepsilon \in(0,1)$, we define $C \circ \boldsymbol{r}$ as a rational $\boldsymbol{r}^{C} \in \mathcal{R}$ such that:

$$
\boldsymbol{r}_{i}^{C}\left(\varepsilon, s_{i}\right)=a_{0}\left(s_{i}\right)+\frac{\operatorname{Poly}_{i}^{N}\left(\varepsilon, s_{i}\right)}{\operatorname{Poly}_{i}^{D}\left(\varepsilon, s_{i}\right)} C(\varepsilon),
$$

for every player $i \in N$ and sequence $s_{i} \in S_{i}$.

REMARK 8 Note that the transformation in Definition 22 is always well defined, as $\boldsymbol{r}(\varepsilon) \in R$ immediately implies $\boldsymbol{r}^{C}(\varepsilon) \in R$.

Letting $\boldsymbol{\beta}, \boldsymbol{\beta}^{C} \in \mathcal{P}$ be such that $\boldsymbol{\beta}(\varepsilon)$, respectively $\boldsymbol{\beta}^{C}(\varepsilon)$, has $\boldsymbol{r}(\varepsilon)$, respectively $\boldsymbol{r}^{C}(\varepsilon)$, as realization plan, it is a direct consequence of Definition 22 that $\boldsymbol{\beta}$ and $\boldsymbol{\beta}^{C}$ have the same limit points (as $\varepsilon$ goes to zero).

REMARK $9 \lim _{\varepsilon \rightarrow 0^{+}} \boldsymbol{\beta}(\varepsilon)=\lim _{\varepsilon \rightarrow 0^{+}} \boldsymbol{\beta}^{C}(\varepsilon)$.

In the following lemma, we prove that, for any player $i \in N$ and information set $u \in U_{i}$, replacing $\boldsymbol{r}(\varepsilon)$ with $\boldsymbol{r}^{C}(\varepsilon)$ does not affect player $i$ 's optimal strategies in the game following $u$, provided that $\varepsilon$ is sufficiently small.

LEMMA 5 Given a two-player ${ }^{21}$ game in sequence form $\Gamma_{\boldsymbol{S}}$, let:

1. $\boldsymbol{r} \in \mathcal{R}$ be rational;

2. $\varepsilon \mapsto C(\varepsilon)$ be a rational function defined over $\varepsilon \in(0,1)$.

Then, there exists $\bar{\varepsilon} \in(0,1)$ such that, for all $\varepsilon \in(0, \bar{\varepsilon})$, and for any player $i \in N$, information set $u \in U_{i}$, and choice $c \in C_{u}$, it holds:

$$
\begin{aligned}
\max _{\substack{r_{i}^{\prime} \in R_{i}: \\
r_{i}^{\prime}(\sigma(u) c)=1}} G_{i}\left(u, \boldsymbol{r}(\varepsilon) / r_{i}^{\prime}\right)=\max _{\substack{r_{i}^{\prime} \in R_{i}: \\
r_{i}^{\prime}(\sigma(u))=1}} G_{i}\left(u, \boldsymbol{r}(\varepsilon) / r_{i}^{\prime}\right) \Longleftrightarrow \\
\max _{\substack{r_{i}^{\prime} \in R_{i}: \\
r_{i}^{\prime}(\sigma(u) c)=1}} G_{i}\left(u, \boldsymbol{r}^{C}(\varepsilon) / r_{i}^{\prime}\right)=\max _{\substack{r_{i}^{\prime} \in R_{i}: \\
r_{i}^{\prime}(\sigma(u))=1}} G_{i}\left(u, \boldsymbol{r}^{C}(\varepsilon) / r_{i}^{\prime}\right) .
\end{aligned}
$$

\footnotetext{
${ }^{21}$ Let us remark that Lemma 5 holds for two-player games only, a counterexample is available from authors at request.
} 
Proof: Let us fix a player $i \in N$, an information set $u \in U_{i}$, and a choice $c \in C_{u}$. Given that each $\boldsymbol{r}_{i}\left(\varepsilon, s_{i}\right)$ is a function of $\varepsilon$, we can write $G_{i}\left(u, \boldsymbol{r}(\varepsilon) / r_{i}^{\prime}\right)=$ $K_{i}\left(r_{i}^{\prime}\right)+V_{i}\left(\varepsilon, r_{i}^{\prime}\right)$, where $K_{i}\left(r_{i}^{\prime}\right) \in[0,1]$ is the component of $G_{i}\left(u, \boldsymbol{r}(\varepsilon) / r_{i}^{\prime}\right)$ that does not depend on $\varepsilon$, while $V_{i}\left(\varepsilon, r_{i}^{\prime}\right)$ is the part depending on $\varepsilon$. Let us consider the case in which $K_{i}\left(r_{i}^{\prime}\right)>0$ (as the case $K_{i}\left(r_{i}^{\prime}\right)=0$ is trivial). Clearly, there exists $\bar{\varepsilon} \in(0,1)$ such that, for any player $i$, information set $u$, and choice $c$, for all $\varepsilon \in(0, \bar{\varepsilon}), V_{i}\left(\varepsilon, r_{i}^{\prime}\right)$ is smaller than $K_{i}\left(r_{i}^{\prime}\right)$ independently of $r_{i}^{\prime}$. Thus, for all $\varepsilon \in(0, \bar{\varepsilon})$ we have that:

$$
\begin{aligned}
& \max _{\substack{r_{i}^{\prime} \in R_{i}: \\
r_{i}^{\prime}(\sigma(u) c)=1}} K_{i}\left(r_{i}^{\prime}\right)+V_{i}\left(\varepsilon, r_{i}^{\prime}\right)=\max _{\substack{r_{i}^{\prime} \in R_{i}: \\
r_{i}^{\prime}(\sigma(u))=1}} K_{i}\left(r_{i}^{\prime}\right)+V_{i}\left(\varepsilon, r_{i}^{\prime}\right) \Longleftrightarrow \\
& \max _{\substack{r_{i}^{\prime} \in R_{i}: \\
r_{i}^{\prime}(\sigma(u) c)=1}} K_{i}\left(r_{i}^{\prime}\right)=\max _{\substack{r_{i}^{\prime} \in R_{i}: \\
r_{i}^{\prime}(\sigma(u))=1}} K_{i}\left(r_{i}^{\prime}\right) \quad \text { and } \\
& \max _{\substack{r_{i}^{\prime} \in R_{i}: \\
r_{i}^{\prime}(\sigma(u) c)=1}} V_{i}\left(\varepsilon, r_{i}^{\prime}\right)=\max _{\substack{r_{i}^{\prime} \in R_{i}: \\
r_{i}^{\prime}(\sigma(u))=1}} V_{i}\left(\varepsilon, r_{i}^{\prime}\right) .
\end{aligned}
$$

Since there are only two players: $G_{i}\left(u, \boldsymbol{r}^{C}(\varepsilon) / r_{i}^{\prime}\right)=K_{i}\left(r_{i}^{\prime}\right)+V_{i}\left(\varepsilon, r_{i}^{\prime}\right) C(\varepsilon)$. This proves the result.

In the following, we are interested in particular transformations in which the function $\varepsilon \mapsto C(\varepsilon)$ has a specific shape, which allows us to make polynomial any rational $\boldsymbol{r} \in \mathcal{R}$.

DeFINITION 23 Given a game in sequence form $\Gamma_{\boldsymbol{S}}$ and a rational $\boldsymbol{r} \in \mathcal{R}$, we define $\boldsymbol{r}^{p} \in \mathcal{R}$ as $C^{p} \circ \boldsymbol{r}$, where the function $\varepsilon \mapsto C^{p}(\varepsilon)$ has the following expression:

$$
C^{p}(\varepsilon)=\prod_{i \in N} \prod_{s_{i} \in S_{i}} \operatorname{Poly}_{i}^{D}\left(\varepsilon, s_{i}\right)
$$

It is an immediate consequence of Definition 23 that:

REMARK 10 Given a rational $\boldsymbol{r} \in \mathcal{R}, \boldsymbol{r}^{p}$ is polynomial.

Motivating Example Following Definition 23, let $C^{p}(\varepsilon)=(1-\varepsilon)^{4}$, which allows us to obtain $\boldsymbol{r}^{p} \in \mathcal{R}$ such that:

$$
\boldsymbol{r}_{1}^{p}(\varepsilon)=\left[\begin{array}{l}
\boldsymbol{r}_{1}^{p}(\varepsilon, \varnothing)=1 \\
\boldsymbol{r}_{1}^{p}(\varepsilon, A)=1-\varepsilon+3 \varepsilon^{2}-3 \varepsilon^{3}+\varepsilon^{4} \\
\boldsymbol{r}_{1}^{p}(\varepsilon, B)=\varepsilon-3 \varepsilon^{2}+3 \varepsilon^{3}-\varepsilon^{4} \\
\boldsymbol{r}_{1}^{p}(\varepsilon, A C)=1-2 \varepsilon+6 \varepsilon^{2}-6 \varepsilon^{3}+2 \varepsilon^{4} \\
\boldsymbol{r}_{1}^{p}(\varepsilon, A D)=\varepsilon-3 \varepsilon^{2}+3 \varepsilon^{3}-\varepsilon^{4}
\end{array}\right] \quad \text { and } \quad \boldsymbol{r}_{2}^{p}(\varepsilon)=\left[\begin{array}{c}
\boldsymbol{r}_{2}^{p}(\varepsilon, \varnothing)=1 \\
\boldsymbol{r}_{2}^{p}(\varepsilon, a)=\frac{1}{2} \\
\boldsymbol{r}_{2}^{p}(\varepsilon, b)=\frac{1}{2}
\end{array}\right]
$$


Now, we are ready to introduce a second type of transformation. First, given a polynomial $\boldsymbol{r} \in \mathcal{R}$, for every player $i \in N$ and sequence $s_{i} \in S_{i}$, let us define $L_{i}\left(s_{i}\right) \in \mathbb{R}^{d\left(s_{i}\right)+1}$ as the coefficients vector of $\operatorname{Poly}_{i}\left(\varepsilon, s_{i}\right)$, i.e.,

$$
L_{i}\left(s_{i}\right)^{T}=\left[\begin{array}{llll}
a_{0}\left(i, s_{i}\right) & a_{1}\left(i, s_{i}\right) & \ldots & a_{d\left(s_{i}\right)}\left(i, s_{i}\right)
\end{array}\right]^{T} .
$$

Let also $d_{i}=\max _{s_{i} \in S_{i}} d\left(s_{i}\right)$ be the maximum degree of polynomials $\operatorname{Poly}_{i}\left(\varepsilon, s_{i}\right)$.

Next, for every player $i \in N$, we define a matrix containing the coefficients of polynomials defining $\boldsymbol{r}_{i}$, as follows.

Definition 24 Given a polynomial $\boldsymbol{r} \in \mathcal{R}$ and a player $i \in N$, we define the coefficients matrix $Q_{i}$ associated with $\boldsymbol{r}_{i}$ as a matrix of dimensions $\left|S_{i}\right| \times\left(d_{i}+1\right)$ whose rows are the vectors $L_{i}\left(s_{i}\right)^{T}$.

In the following, we use $Q_{i}[x]$ to denote the $x$-th column of matrix $Q_{i}$. Moreover, $Q_{i}[x] \neq \mathbf{0}$ means that $Q_{i}[x]$ is nonzero component wise.

We are now ready to define the transformation.

DeFINITION 25 Given a game in sequence form $\Gamma_{\boldsymbol{S}}$ and a polynomial $\boldsymbol{r} \in \mathcal{R}$, we define a shift of $\boldsymbol{r}$, written $\operatorname{Shift}(\boldsymbol{r})$, as a polynomial $\boldsymbol{r}^{s} \in R$ such that, for every player $i \in N$, the coefficients matrix $Q_{i}^{s}$ associated with $\boldsymbol{r}_{i}^{s}$ is a matrix of dimensions $\left|S_{i}\right| \times\left(d_{i}^{s}+1\right)$ that satisfies the following conditions:

1. $Q_{i}[0]=Q_{i}^{s}[0]$;

2. for all $x \in\left\{1, \ldots, d_{i}\right\}$ such that $Q_{i}[x] \neq \mathbf{0}$, there exists $s_{i}(x) \in\left\{1, \ldots, d_{i}^{s}\right\}$ such that $Q_{i}[x]=Q_{i}^{s}\left[s_{i}(x)\right]$;

3. for all $x, y \in\left\{1, \ldots, d_{i}\right\}$ with $x<y$ such that $Q_{i}[x] \neq \mathbf{0}$ and $Q_{i}[y] \neq \mathbf{0}$, we have that $s_{i}(x)<s_{i}(y)$.

In words, for every player $i \in N$, Shift $(\boldsymbol{r})$ modifies the shape of the polynomials defining sequence probabilities $\boldsymbol{r}_{i}\left(\varepsilon, s_{i}\right)$ in a way that preserves their ordering for sufficiently small values of $\varepsilon$. The idea is that the resulting polynomials have the same coefficients of the original ones, but the degrees are changed so that if two coefficients were multiplied by the same power of $\varepsilon$ in the original polynomials, then the same holds in the new ones.

REMARK 11 Let us remark that, given the coefficients matrices $Q_{i}^{s}$ obtained by applying a shift operation to a polynomial $\boldsymbol{r} \in \mathcal{R}$, we can easily recover their associated polynomial $\boldsymbol{r}^{s} \in \mathcal{R}$.

Letting $\boldsymbol{\beta}, \boldsymbol{\beta}^{s} \in \mathcal{P}$ be such that $\boldsymbol{\beta}(\varepsilon)$, respectively $\boldsymbol{\beta}^{s}(\varepsilon)$, has $\boldsymbol{r}(\varepsilon)$, respectively $\boldsymbol{r}^{s}(\varepsilon)$, as realization plan, it is a direct consequence of Definition 25 that:

REMARK $12 \lim _{\varepsilon \rightarrow 0^{+}} \boldsymbol{\beta}(\varepsilon)=\lim _{\varepsilon \rightarrow 0^{+}} \boldsymbol{\beta}^{s}(\varepsilon)$. 
Unfortunately, a generic shift transformation does not preserve player $i$ 's optimal strategies at the information sets. In order to guarantee this, we need to introduce particular shift transformations.

DEFINITION 26 Given a game in sequence form $\Gamma_{\boldsymbol{S}}$, a polynomial $\boldsymbol{r} \in \mathcal{R}$, and $s \in \mathbb{N}$, we define a proportional shift of $\boldsymbol{r}$ with degree $s$, written $s$-Shift $(\boldsymbol{r})$, as a particular shift such that, for every player $i \in N$ and $x \in\left\{1, \ldots, d_{i}\right\}$ with $Q_{i}[x] \neq \mathbf{0}$, it holds $s_{i}(x)=x s-x$.

REMARK 13 Note that $\boldsymbol{r}^{s}(\varepsilon)$ can be obtained from $\boldsymbol{r} \in \mathcal{R}$ by substituting each appearance of $\varepsilon$ with $\varepsilon^{s}$, i.e., it is the case that $\boldsymbol{r}^{s}(\varepsilon)=\boldsymbol{r}\left(\varepsilon^{s}\right)$.

We can prove the following fundamental property of proportional shifts.

LEMMA 6 Given a game in sequence form $\Gamma_{\boldsymbol{S}}$ and a polynomial $\boldsymbol{r} \in \mathcal{R}$, there exists $\bar{\varepsilon} \in(0,1)$ such that, for all $\varepsilon \in(0, \bar{\varepsilon})$, and for any player $i \in N$, information set $u \in u$, and choice $c \in C_{u}$, it holds:

$$
\begin{aligned}
& \max _{\substack{r_{i}^{\prime} \in R_{i}: \\
r_{i}^{\prime}(\sigma(u) c)=1}} G_{i}\left(u, \boldsymbol{r}(\varepsilon) / r_{i}^{\prime}\right)=\max _{\substack{r_{i}^{\prime} \in R_{i}: \\
r_{i}^{\prime}(\sigma(u))=1}} G_{i}\left(u, \boldsymbol{r}(\varepsilon) / r_{i}^{\prime}\right) \Longleftrightarrow \\
& \max _{\substack{r_{i}^{\prime} \in R_{i}: \\
r_{i}^{\prime}(\sigma(u) c)=1}} G_{i}\left(u, \boldsymbol{r}^{s}(\varepsilon) / r_{i}^{\prime}\right)=\max _{\substack{r_{i}^{\prime} \in R_{i}: \\
r_{i}^{\prime}(\sigma(u))=1}} G_{i}\left(u, \boldsymbol{r}^{s}(\varepsilon) / r_{i}^{\prime}\right),
\end{aligned}
$$

where $\boldsymbol{r}^{s} \in \mathcal{R}$ is defined as $s$-Shift $(\boldsymbol{r})$, for some $s \in \mathbb{N}$.

ProOF: Let us fix a player $i \in N$, an information set $u \in U_{i}$, and a choice $c \in C_{u}$. Given that each $\boldsymbol{r}_{i}\left(\varepsilon, s_{i}\right)$ is a function of $\varepsilon$, we can write $G_{i}\left(u, \boldsymbol{r}(\varepsilon) / r_{i}^{\prime}\right)=$ $K_{i}\left(r_{i}^{\prime}\right)+V_{i}\left(\varepsilon, r_{i}^{\prime}\right)$, where $K_{i}\left(r_{i}^{\prime}\right) \in[0,1]$ is the component of $G_{i}\left(u, \boldsymbol{r}(\varepsilon) / r_{i}^{\prime}\right)$ that does not depend on $\varepsilon$, while $V_{i}\left(\varepsilon, r_{i}^{\prime}\right)$ is the part depending on $\varepsilon$. Let us consider the case in which $K_{i}\left(r_{i}^{\prime}\right)>0$ (as the case $K_{i}\left(r_{i}^{\prime}\right)=0$ is trivial). Clearly, there exists $\bar{\varepsilon} \in(0,1)$ such that, for any player $i$, information set $u$, and choice $c$, for all $\varepsilon \in(0, \bar{\varepsilon}), V_{i}\left(\varepsilon, r_{i}^{\prime}\right)$ is smaller than $K_{i}\left(r_{i}^{\prime}\right)$ independently of $r_{i}^{\prime}$. Thus, we can write the following:

$$
\begin{gathered}
\max _{\substack{r_{i}^{\prime} \in R_{i}: \\
r_{i}^{\prime}(\sigma(u) c)=1}} K_{i}\left(r_{i}^{\prime}\right)+V_{i}\left(\varepsilon, r_{i}^{\prime}\right)=\max _{\substack{r_{i}^{\prime} \in R_{i}: \\
r_{i}^{\prime}(\sigma(u))=1}} K_{i}\left(r_{i}^{\prime}\right)+V_{i}\left(\varepsilon, r_{i}^{\prime}\right) \Longleftrightarrow \\
\max _{\substack{r_{i}^{\prime} \in R_{i}: \\
r_{i}^{\prime}(\sigma(u) c)=1}} K_{i}\left(r_{i}^{\prime}\right)=\max _{\substack{r_{i}^{\prime} \in R_{i}: \\
r_{i}^{\prime}(\sigma(u))=1}} K_{i}\left(r_{i}^{\prime}\right) \quad \text { and } \\
\max _{\substack{r_{i}^{\prime} \in R_{i} \\
r_{i}^{\prime}(\sigma(u) c)=1}} V_{i}\left(\varepsilon, r_{i}^{\prime}\right)=\max _{\substack{r_{i}^{\prime} \in R_{i}: \\
r_{i}^{\prime}(\sigma(u))=1}} V_{i}\left(\varepsilon, r_{i}^{\prime}\right) .
\end{gathered}
$$

By definition, we have: $G_{i}\left(u, \boldsymbol{r}^{\boldsymbol{s}}(\varepsilon) / r_{i}^{\prime}\right)=K_{i}\left(r_{i}^{\prime}\right)+V_{i}\left(\varepsilon^{s}, r_{i}^{\prime}\right)$, which concludes the proof.

Q.E.D. 
In the rest of this work, we are interested in a particular proportional shift transformation in which $s$ is equal to the maximum length of all players' sequences.

DEFINITION 27 Given a game in sequence form $\Gamma_{\boldsymbol{S}}$ and a rational $\boldsymbol{r} \in \mathcal{R}$, we define $\boldsymbol{r}^{*} \in \mathcal{R}$ as the result of a proportional shift $s^{\max }$-Shift $\left(\boldsymbol{r}^{p}\right)$, where we have $s^{\max }=\max _{i \in N, s_{i} \in S_{i}}\left|s_{i}\right|$.

Motivating Example Following Definition 2\%, we can define $\boldsymbol{r}^{*} \in \mathcal{R}$. First, we report below the coefficients matrix $Q_{1}$ associated with $\boldsymbol{r}_{1}^{p}$.

\begin{tabular}{r|rrrrr}
$x$ & 0 & 1 & 2 & 3 & 4 \\
\hline$\varnothing$ & 1 & 0 & 0 & 0 & 0 \\
$A$ & 1 & -1 & 3 & -3 & 1 \\
$B$ & 0 & 1 & -3 & 3 & -1 \\
$A C$ & 1 & -2 & 6 & -6 & 2 \\
$A D$ & 0 & 1 & -3 & 3 & -1
\end{tabular}

Then, we apply an $s^{\max }{ }_{-} \operatorname{Shift}\left(\boldsymbol{r}^{p}\right)$ transformation, with $s^{\max }=2$, which allows us to obtain the following coefficients matrix $Q_{1}^{s}$.

\begin{tabular}{r|rrrrrrrrr}
$x$ & 0 & 1 & 2 & 3 & 4 & 5 & 6 & 7 & 8 \\
\hline$\varnothing$ & 1 & 0 & 0 & 0 & 0 & 0 & 0 & 0 & 0 \\
$A$ & 1 & 0 & -1 & 0 & 3 & 0 & -3 & 0 & 1 \\
$B$ & 0 & 0 & 1 & 0 & -3 & 0 & 3 & 0 & -1 \\
$A C$ & 1 & 0 & -2 & 0 & 6 & 0 & -6 & 0 & 2 \\
$A D$ & 0 & 0 & 1 & 0 & -3 & 0 & 3 & 0 & -1
\end{tabular}

Thus, $\boldsymbol{r}^{*}$ is defined as follows:

$$
\boldsymbol{r}_{1}^{*}(\varepsilon)=\left[\begin{array}{l}
\boldsymbol{r}_{1}^{*}(\varepsilon, \varnothing)=1 \\
\boldsymbol{r}_{1}^{*}(\varepsilon, A)=1-\varepsilon^{2}+3 \varepsilon^{4}-3 \varepsilon^{6}+\varepsilon^{8} \\
\boldsymbol{r}_{1}^{*}(\varepsilon, B)=\varepsilon^{2}-3 \varepsilon^{4}+3 \varepsilon^{6}-\varepsilon^{8} \\
\boldsymbol{r}_{1}^{*}(\varepsilon, A C)=1-2 \varepsilon^{2}+6 \varepsilon^{4}-6 \varepsilon^{6}+2 \varepsilon^{8} \\
\boldsymbol{r}_{1}^{*}(\varepsilon, A D)=\varepsilon^{2}-3 \varepsilon^{4}+3 \varepsilon^{6}-\varepsilon^{8}
\end{array}\right] \quad \text { and } \quad \boldsymbol{r}_{2}^{*}(\varepsilon)=\left[\begin{array}{l}
\boldsymbol{r}_{2}^{*}(\varepsilon, \varnothing)=1 \\
\boldsymbol{r}_{2}^{*}(\varepsilon, a)=\frac{1}{2} \\
\boldsymbol{r}_{2}^{*}(\varepsilon, b)=\frac{1}{2}
\end{array}\right] .
$$

\subsection{Perturbed Sequence Form}

Now, suppose we are given a quasi-perfect equilibrium $\beta \in B$ of a game in extensive form $\Gamma$, obtained for some completely mixed and rational $\boldsymbol{\beta} \in \mathcal{P}$. Let $\boldsymbol{r} \in \mathcal{R}$ be such that $\boldsymbol{r}(\varepsilon)$ is the realization plan of $\boldsymbol{\beta}(\varepsilon)$. In the following, let us define $\boldsymbol{r}^{*} \in \mathcal{R}$ as in Definition 27, and let $\boldsymbol{\beta}^{*} \in \mathcal{P}$ be such that $\boldsymbol{\beta}^{*}(\varepsilon)$ has $\boldsymbol{r}^{*}(\varepsilon)$ as realization plan. For ease of presentation, given $\beta \in B$, for every player $i \in N$ and sequence $s_{i} \in S_{i}$, we say that $s_{i} \in \mathcal{S}(\beta)$ if and only if $\beta_{i}(c)>0$, where $c$ is the last choice in sequence $s_{i}$, i.e., $\mathcal{S}(\beta)$ represents the support of $\beta$, which is the set of sequences whose last choice is played with positive probability in $\beta$. 
DeFINITION 28 Given a quasi-perfect equilibrium $\beta \in B$ of a game in extensive form $\Gamma$, we define its associated perturbed sequence form $\left(\Gamma_{\boldsymbol{S}},\left\{\ell_{i}^{\beta}\right\}_{i \in N}\right)$ such that, for any player $i \in N$ and sequence $s_{i} \in S_{i}$ :

- if $s_{i} \in \mathcal{S}(\beta)$, then $\ell_{i}^{\beta}\left(\varepsilon, s_{i}\right)=\varepsilon^{d_{\min }\left(s_{i}^{\prime}\right)+\left|s_{i}\right|-\left|s_{i}^{\prime}\right|}$, where $s_{i}^{\prime} \in S_{i}$ is the longest sequence such that $s_{i}^{\prime} \sqsubset s_{i}$ and $s_{i}^{\prime} \notin \mathcal{S}(\beta)$, while $d_{\min }\left(s_{i}^{\prime}\right)$ is the minimum degree of $\varepsilon$ in the polynomial defining $\boldsymbol{r}_{i}^{*}\left(\varepsilon, s_{i}^{\prime}\right)$;

- if $s_{i} \notin \mathcal{S}(\beta)$, then $\ell_{i}^{\beta}\left(\varepsilon, s_{i}\right)=\boldsymbol{r}_{i}^{*}\left(\varepsilon, s_{i}\right)$.

REMARK 14 Note that, given Definition 28 and how the transformations in the previous sections are performed, it is always the case that each $\ell_{i}^{\beta}$ is well defined and satisfies the conditions of Definition 19. Thus, the perturbed sequence form $\left(\Gamma_{\boldsymbol{S}},\left\{\ell_{i}^{\beta}\right\}_{i \in N}\right)$ is always well defined.

Motivating Example Let us return to our motivating example and show how the perturbed sequence form $\left(\Gamma_{\boldsymbol{S}},\left\{\ell_{i}^{\beta}\right\}_{i \in N}\right)$ is defined. Given the definition of $\beta \in B$, we have $\mathcal{S}(\beta)=\{A, A C, a, b\}$ and, thus:

$\ell_{1}(\varepsilon)=\left[\begin{array}{l}\ell_{1}(\varepsilon, \varnothing)=1 \\ \ell_{1}(\varepsilon, A)=\varepsilon \\ \ell_{1}(\varepsilon, B)=\varepsilon^{2}-3 \varepsilon^{4}+3 \varepsilon^{6}-\varepsilon^{8} \\ \ell_{1}(\varepsilon, A C)=\varepsilon^{2} \\ \ell_{1}(\varepsilon, A D)=\varepsilon^{2}-3 \varepsilon^{4}+3 \varepsilon^{6}-\varepsilon^{8}\end{array}\right] \quad$ and $\quad \ell_{2}(\varepsilon)=\left[\begin{array}{c}\ell_{2}(\varepsilon, \varnothing)=1 \\ \ell_{2}(\varepsilon, a)=\varepsilon \\ \ell_{2}(\varepsilon, b)=\varepsilon\end{array}\right]$.

It is easy to check that each realization plan profile $\boldsymbol{r}^{*}(\varepsilon)$ is a Nash equilibrium of $\Gamma_{\boldsymbol{S}}(\varepsilon)$, provided that $\varepsilon$ is sufficiently small.

TheOrem 3 Given a quasi-perfect equilibrium $\beta \in B$ of a game in extensive form $\Gamma$, let $\left(\Gamma_{\boldsymbol{S}},\left\{\ell_{i}^{\beta}\right\}_{i \in N}\right)$ be its associated perturbed sequence form. Then, $\boldsymbol{r}^{*}(\varepsilon)$ defines a sequence of Nash equilibria of perturbed games $\Gamma_{\boldsymbol{S}}(\varepsilon)$ and $\beta$ is a limit point (as $\varepsilon$ goes to zero) of $\boldsymbol{\beta}^{*}(\varepsilon)$.

Proof: Let us consider an $\bar{\varepsilon} \in(0,1)$ such that, for all $\varepsilon \in(0,1), \boldsymbol{r}^{*}(\varepsilon)$ is a well-defined realization plan profile of $\Gamma_{\boldsymbol{S}}(\varepsilon)$ and Lemmas 5 and 6 hold. Clearly, given Remarks 9 and 12, we have that $\beta$ is a limit point (as $\varepsilon$ goes to zero) of $\boldsymbol{\beta}^{*}(\varepsilon)$. Moreover, given Definition 28, we have that, for every player $i \in N$, information set $u \in U_{i}$, and choice $c \in \sigma(u)$, if $\beta_{i}(c)=0$, then $\boldsymbol{r}^{*}\left(\varepsilon_{k}, \sigma(u) c\right)=$ $\ell_{i}^{\beta}(\varepsilon, \sigma(u) c)$, while, whenever $\beta_{i}(c)>0$, we have $\boldsymbol{r}^{*}(\varepsilon, \sigma(u) c)>\ell_{i}^{\beta}(\varepsilon, \sigma(u) c)$. Invoking Lemma 4 and Theorem 1 allows us to conclude that $\boldsymbol{r}^{*}(\varepsilon)$ is a Nash equilibrium of $\Gamma_{\boldsymbol{S}}(\varepsilon)$.

Q.E.D.

\section{CONCLUSION}

The main contribution of this paper is the characterization of quasi-perfect equilibria of two-player games as limit points of sequences of Nash equilibria of 
a particular class of perturbed games in sequence form. This result is formalized in Theorems 2 and 3.

Beside the characterization of quasi-perfect equilibria for two-player games, the results of this paper show that the sequence form defined by Von Stengel [1996] is not merely a computationally efficient representation of a game, but it also plays a crucial role when defining the properties of the solution concepts. In particular, as shown in Theorem 1 and Lemma 4, our perturbed games in sequence form allow us to capture game features that cannot be expressed using the extensive form, such as the idea of playing optimally against opponents' trembles in the game following an information set, which is the core idea behind quasi-perfection. More precisely, while extensive-form perfect equilibria are the limit points of Nash equilibria of perturbed games in extensive form, for any vanishing perturbation applied to players' choices, quasi-perfect equilibria are the limit points of Nash equilibria of perturbed games in sequence form, once a specific class of vanishing perturbations is applied to players' sequences.

Interestingly, as discussed in Section 3.3, we can map perturbations defined on the extensive form to associated perturbations applied to the sequence form, and vice versa. However, this mapping returns perturbations that depend also on the strategies themselves and not only on the perturbation magnitude. This means that quasi-perfect equilibria are also the limit points of Nash equilibria of perturbed games in extensive form, when the perturbation applied to a player's choice depends on the player's behavioral strategy for previous choices in the game tree. We recall that an analogous result holds for extensive-form perfect equilibria: they are the limit points of Nash equilibria of perturbed games in sequence form, when the perturbation applied to a player's sequence depends on the player's realization plan. Thus, even if the sequence form and the extensive form are equivalent, the extensive form is the most natural form when dealing with extensive-form perfect equilibria, while the sequence form is the most natural form when dealing with quasi-perfect equilibria. We remark that the sequence form is superior to the extensive form for any computational task.

A final interesting aspect is that Theorem 3, once again, shows the marked difference between refinements in two-player and $n$-player games. In particular, Lemma 5 cannot be easily extended to general $n$-player games. However, this lemma is necessary only when the quasi-perfect equilibrium of interest can only be obtained for a sequence of completely mixed behavior strategy profiles described by rational functions in $\varepsilon$. Therefore, for $n$-player games a weaker characterization holds, which only encompasses quasi-perfect equilibria that are obtainable with sequences of completely mixed behavior strategy profiles described by polynomial functions in $\varepsilon$.

\section{REFERENCES}

Lawrence Blume, Adam Brandenburger, and Eddie Dekel. Lexicographic probabilities and equilibrium refinements. Econometrica: Journal of the Econometric Society, pages 81-98, 
1991.

Lawrence E Blume and William R Zame. The algebraic geometry of perfect and sequential equilibrium. Econometrica: Journal of the Econometric Society, pages 783-794, 1994.

Gabriele Farina and Nicola Gatti. Extensive-form perfect equilibrium computation in twoplayer games. In Proceedings of the Thirty-First AAAI Conference on Artificial Intelligence, February 4-9, 2017, San Francisco, California, USA., pages 502-508, 2017.

Srihari Govindan and Tilman Klumpp. Perfect equilibrium and lexicographic beliefs. International Journal of Game Theory, 31(2):229-243, 2003.

Srihari Govindan and Robert Wilson. Axiomatic equilibrium selection for generic two-player games. Econometrica, 80(4):1639-1699, 2012.

Srihari Govindan and Robert B Wilson. Sufficient conditions for stable equilibria. Theoretical Economics, 1(2):167-206, 2006.

Peter J Hammond. Elementary non-archimedean representations of probability for decision theory and games. In Patrick Suppes: scientific philosopher, pages 25-61. Springer, 1994.

J. Hillas, T. Kao, and A. Schiff. A real algebraic proof of the generic equivalence of quasi-perfect and sequential equilibria. unpublished, 2016.

John Hillas and Elon Kohlberg. Foundations of strategic equilibrium. Handbook of Game Theory with Economic Applications, 3:1597-1663, 2002.

Elon Kohlberg and Philip J Reny. Independence on relative probability spaces and consistent assessments in game trees. Journal of Economic Theory, 75(2):280-313, 1997.

David M Kreps and Robert Wilson. Sequential equilibria. Econometrica: Journal of the Econometric Society, pages 863-894, 1982.

HW Kuhn. Extensive games and the problem of information, contributions to the theory of games ii. Annals of Mathematics Studies, 28:193-216, 1953.

Andrew McLennan. Consistent conditional systems in noncooperative game theory. International Journal of Game Theory, 18(2):141-174, 1989.

J-F Mertens. Two examples of strategic equilibrium. Games and Economic Behavior, 8(2): 378-388, 1995.

Peter Bro Miltersen and Troels Bjerre Sørensen. Computing a quasi-perfect equilibrium of a two-player game. Economic Theory, 42(1):175-192, 2010.

Roger B Myerson. Multistage games with communication. Econometrica: Journal of the Econometric Society, pages 323-358, 1986.

Carlos Pimienta and Jianfei Shen. On the equivalence between (quasi-) perfect and sequential equilibria. International Journal of Game Theory, 43(2):395-402, 2014

Reinhard Selten. Reexamination of the perfectness concept for equilibrium points in extensive games. International journal of game theory, 4(1):25-55, 1975.

Eric Van Damme. A relation between perfect equilibria in extensive form games and proper equilibria in normal form games. International Journal of Game Theory, 13(1):1-13, 1984.

Bernhard Von Stengel. Efficient computation of behavior strategies. Games and Economic Behavior, 14(2):220-246, 1996.

Bernhard Von Stengel and Françoise Forges. Extensive-form correlated equilibrium: Definition and computational complexity. Mathematics of Operations Research, 33(4):1002-1022, 2008. 